\title{
Elemental Abundances of Kepler Objects of Interest in APOGEE. I. Two Distinct Orbital Period Regimes Inferred from Host Star Iron Abundances
}

\author{
Robert F. Wilson ${ }^{1}$, Johanna Teske ${ }^{2,13}$, Steven R. Majewski ${ }^{1}$ (i), Katia Cunha ${ }^{3,4}$, Verne Smith ${ }^{5}$, Diogo Souto ${ }^{3}$, Chad Bender ${ }^{4}$,

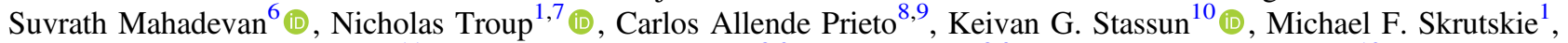 \\ Andrés Almeida ${ }^{11}$, D. A. García-Hernández ${ }^{8,9}$, Olga Zamora ${ }^{8,9}$, and Jonathan Brinkmann ${ }^{12}$ \\ ${ }^{1}$ Department of Astronomy, University of Virginia, Charlottesville, VA 22904-4325, USA \\ ${ }^{2}$ Observatories of the Carnegie Institution for Science, 813 Santa Barbara St., Pasadena, CA 91101, USA \\ ${ }^{3}$ Observatório Nacional, Rua General José Cristino, 77, 20921-400 São Cristóvão, Rio de Janeiro, RJ, Brazil \\ ${ }^{4}$ Steward Observatory, University of Arizona, 933 North Cherry Avenue, Tucson, AZ 85721-0065, USA \\ ${ }^{5}$ National Optical Astronomy Observatory, 950 North Cherry Avenue, Tucson, AZ 85719, USA \\ ${ }^{6}$ Department of Astronomy \& Astrophysics, Pennsylvania State University, University Park, PA 16802, USA \\ ${ }^{7}$ Department of Physics, Salisbury University, Salisbury, MD 21801-6860, USA \\ ${ }^{8}$ Instituto de Astrofísica de Canarias, E-38205 La Laguna, Tenerife, Spain \\ ${ }^{9}$ Departamento de Astrofísica, Universidad de La Laguna, E-38206 La Laguna, Tenerife, Spain \\ ${ }^{10}$ Department of Physics and Astronomy, Vanderbilt University, VU Station 1807, Nashville, TN 37235, USA \\ ${ }^{11}$ Instituto de Investigación Multidisciplinario en Ciencia y Tecnología, Universidad de La Serena, Benavente 980, La Serena, Chile \\ ${ }^{12}$ Apache Point Observatory, P.O. Box 59, Sunspot, NM 88349, USA \\ Received 2017 September 28; revised 2017 November 28; accepted 2017 December 1; published 2018 January 18
}

\begin{abstract}
The Apache Point Observatory Galactic Evolution Experiment (APOGEE) has observed $\sim 600$ transiting exoplanets and exoplanet candidates from Kepler (Kepler Objects of Interest, KOIs), most with $\geqslant 18$ epochs. The combined multi-epoch spectra are of high signal-to-noise ratio (typically $\geqslant 100$ ) and yield precise stellar parameters and chemical abundances. We first confirm the ability of the APOGEE abundance pipeline, ASPCAP, to derive reliable $[\mathrm{Fe} / \mathrm{H}]$ and effective temperatures for FGK dwarf stars-the primary Kepler host stellar type-by comparing the ASPCAP-derived stellar parameters with those from independent high-resolution spectroscopic characterizations for 221 dwarf stars in the literature. With a sample of 282 close-in $(P<100$ days $)$ KOIs observed in the APOGEE KOI goal program, we find a correlation between orbital period and host star [Fe/H] characterized by a critical period, $P_{\text {crit }}=8.3_{-4.1}^{+0.1}$ days, below which small exoplanets orbit statistically more metalenriched host stars. This effect may trace a metallicity dependence of the protoplanetary disk inner radius at the time of planet formation or may be a result of rocky planet ingestion driven by inward planetary migration. We also consider that this may trace a metallicity dependence of the dust sublimation radius, but we find no statistically significant correlation with host $T_{\text {eff }}$ and orbital period to support such a claim.
\end{abstract}

Key words: planetary systems - planets and satellites: formation - stars: abundances

Supporting material: machine-readable tables

\section{Introduction}

With the advent of the Kepler mission (Borucki et al. 2010; Koch et al. 2010; Borucki 2016), statistical studies of exoplanets, particularly small planets $\left(R_{p} \lesssim 4 R_{\oplus}\right)$, have become possible. While a key finding of such studies is that small planets are common in the Galaxy in general (e.g., Howard et al. 2012; Dressing \& Charbonneau 2013; Petigura et al. 2013; Batalha 2014; Burke et al. 2015; Silburt et al. 2015), distinguishing the characteristics of these planets and how they may relate to the properties of their host stars is of interest from formation and detection perspectives. From population studies of larger planets detected by the radial velocity (RV) method, it was clear early on that host star metallicity ${ }^{14}$ was related to the frequency at which these planets form (Gonzalez 1998; Heiter \& Luck 2003; Santos et al. 2004; Valenti \& Fischer 2005), a trend that appears to decrease in strength with decreasing planet mass and/or radius (e.g., Sousa et al. 2008; Ghezzi et al. 2010; Schlaufman \&

\footnotetext{
${ }^{13}$ Carnegie Origins Fellow, jointly appointed by Carnegie DTM and Observatories.

${ }^{14}$ Usually parameterized by the number density of iron nuclei in a star's photosphere relative to the amount of hydrogen, normalized to these values in the Sun: $[\mathrm{Fe} / \mathrm{H}]$, where $[\mathrm{X} / \mathrm{H}]=\log \left(N_{\mathrm{X}} / N_{\mathrm{H}}\right)-\log \left(N_{\mathrm{X}} / N_{\mathrm{H}}\right)_{\text {solar }}$.
}

Laughlin 2011; Buchhave et al. 2012; Buchhave \& Latham 2015; Wang \& Fischer 2015). Now, the most prevalent explanation of this trend is that it is evidence of the core accretion method of planet formation (e.g., Rice \& Armitage 2003; Ida \& Lin 2004; Alibert et al. 2011; Mordasini et al. 2012a) and that host star metallicity is a proxy for the surface density of the solid material in a protoplanetary disk; higher solid surface densities facilitate the faster growth of the solid cores of larger planets, giving them more time to accrete gaseous envelopes.

In addition to the trend between host star $[\mathrm{Fe} / \mathrm{H}]$ and the frequency of different types of planets, other relationships between stellar metallicity and planet properties have also come to light. For instance, Dawson \& Murray-Clay (2013) used the evidence that giant planets orbiting $[\mathrm{Fe} / \mathrm{H}]<0$ stars generally have lower-eccentricity orbits to suggest that planet-planet scattering is the dominant mechanism for inward migration of giant planets, since higher-[Fe/H] systems are more likely to form multiple, closely spaced giant planets. Buchhave et al. (2014) analyzed ground-based optical spectra of Kepler Objects of Interest (KOIs) to measure spectroscopic metallicities and found three regimes of exoplanet sizes, split at $R_{p} \sim 1.7 R_{\oplus}$ and $R_{p} \sim 3.9 R_{\oplus}$, distinguished by different (increasing with $R_{p}$ ) host star metallicities. Using the same 
data but with a more rigorous statistical analysis, Schlaufman (2015) instead favored a single, continuous relationship between planet radius and stellar metallicity. Interestingly, recent results show that (i) $2-6 R_{\oplus}$ planets with orbital periods from 1 to 10 days ("hot Neptunes") show an increase in host star $[\mathrm{Fe} / \mathrm{H}]$ compared to typical planet-hosting stars, similar to that of hot Jupiter $\left(R_{p} \geqslant 10 R_{\oplus}\right)$ planets (Dong et al. 2017), and (ii) at $<1$ day orbital periods, $\leqslant 2 R_{\oplus}$ planet host stars have significantly different metallicities than hot Jupiter host stars but similar metallicities to stars hosting $2-4 R_{\oplus}$ planets with 1-10 day periods (Winn et al. 2017). These studies exemplify the (evidently) intricate relationship between the metallicities of host stars and the sizes and orbital configurations of the planets that form around them.

We intend to further characterize this intricate relationship by investigating how planet orbital period is tied to host star metallicity. This is a topic that has recently been explored by several other studies.

Beaugé \& Nesvorný (2013) examined both confirmed exoplanet systems and Kepler candidate multiple-planet systems to show (i) a lack of small $\left(R_{p} \lesssim 4 R_{\oplus}\right)$, short-period $(P<5$ days) planets around low-metallicity (bulk $[\mathrm{m} / \mathrm{H}]<$ -0.2 dex, from Buchhave et al. 2012) stars, and (ii) a dearth of 4-8 $R_{\oplus}$ planets at $P \leqslant 100$ days around low-metallicity stars. At the time, trends also held in the planetary mass versus period plane; for example, $M_{p} \sin i<0.05 M_{\mathrm{Jup}}$ planets in short orbits were not found around $[\mathrm{Fe} / \mathrm{H}]<-0.2$ dex stars, and planets between the masses of Neptune and Saturn with $P \leqslant 100$ days were mostly absent around $[\mathrm{Fe} / \mathrm{H}]<-0.2$ dex stars. The authors explained these observed trends with delayed formation and less planetary migration in metal-depleted protoplanetary disks. We note that the trends in Beaugé \& Nesvorný (2013) are slightly reduced in significance when more up-to-date planet samples are considered (see Dawson et al. 2015, discussed below).

Similarly, Adibekyan et al. (2013) found from the HARPS GTO RV survey (Mayor et al. 2003; Lo Curto et al. 2010; Santos et al. 2011) that $\sim 0.03-4 M_{\text {Jup }}$ planets orbiting stars with $[\mathrm{Fe} / \mathrm{H}]<-0.1$ dex have longer periods than same-mass planets orbiting stars with $[\mathrm{Fe} / \mathrm{H}]>-0.1$ dex. Specifically, Adibekyan et al. find that all $M_{p} \sin i<0.03 M_{\text {Jup }}$ planets orbiting $[\mathrm{Fe} / \mathrm{H}]>-0.1$ dex stars have periods $<18$ days, and they also suggest that smaller planets orbiting more metal-rich stars are more likely to migrate toward or form close to their host stars compared to planets orbiting more metal-poor stars.

Dawson et al. (2015) explored a theoretical framework motivated by these observational trends, combining analytical estimates for the formation of planetary embryos (which merge to form super-Earths and the cores of mini-Neptunes) with numerical simulations of atmospheric accretion in disks having varying solid surface densities. Interpreting their model predictions in the context of easily observed quantities (planet radius and host star metallicity), Dawson et al. find that disks with high solid surface density (metallicity) generate $2 M_{\oplus}$ cores before the gas disk dissipates ( $\sim 1 \mathrm{Myr})$, which enables the cores to more readily accrete significant atmospheres and thus increase their gas to rock fraction $\left(R_{p}\right)$. Furthermore, these authors find a match with current observations-i.e., that metalrich stars lack rock-dominated $\left(R_{p}<1.5 R_{\oplus}\right)$ planets beyond $\sim 15$ day periods - and suggest that this may indicate that embryo, and thus final core, masses grow faster at larger orbital distances in metal-rich versus metal-poor disks, thus producing gas-enveloped, larger- $R_{p}$ planets.

Most recently, Mulders et al. (2016) used over 20,000 stars observed by both Kepler and LAMOST (Cui et al. 2012) to confirm that short-period planets $(\lesssim 10$ days $)$ are preferentially found around more metal-rich stars $([\mathrm{Fe} / \mathrm{H}] \simeq 0.15 \pm 0.05 \mathrm{dex})$, whereas longer-period planets orbit roughly solar metallicity $([\mathrm{Fe} / \mathrm{H}] \sim 0)$ host stars. In the $P<10$ day sample, it is the smallest-radius planets $\left(<1.7 R_{\oplus}\right)$ that have the largest host $[\mathrm{Fe} / \mathrm{H}]$ contrast compared to their similarly sized but longerperiod counterparts, with an occurrence-weighted $\Delta[\mathrm{Fe} / \mathrm{H}] \simeq$ $0.25 \pm 0.07$. Mulders et al. suggest that their results may be evidence that the inner edges of protoplanetary disks around more metal-rich stars are closer in than around more metal-depleted stars. The trend observed by Mulders et al. is in contrast to the assessment by Winn et al. (2017), who comment that their metallicities (from Petigura et al. 2017, using HIRES/Keck data from the California Kepler Survey) of small planet host stars show no such period dependence. Differences in sample selection may influence the differences in the Mulders et al. versus Winn et al. results.

Many of the works above use moderate- to high-resolution optical spectroscopy to derive host star parameters. Indeed, the original Kepler Input Catalog (KIC) was not intended for detailed studies of host star metallicity (Brown et al. 2011), which motivated numerous follow-up spectroscopic campaigns to better characterize KOIs (e.g., Bruntt et al. 2012; Buchhave et al. 2012, 2014; Everett et al. 2013; Dong et al. 2014; Brewer et al. 2016; Petigura et al. 2017). In this work we present a study of host star $[\mathrm{Fe} / \mathrm{H}]$ versus planetary orbital period using high-resolution near-infrared spectroscopy of KOIs taken by the Sloan Digital Sky Survey's Apache Point Observatory Galactic Evolution Experiment (APOGEE; Majewski et al. 2017). In Section 2 we discuss the APOGEE stellar parameter derivation and validate the $[\mathrm{Fe} / \mathrm{H}]$ and $T_{\text {eff }}$ values produced by APOGEE's automated stellar parameter pipeline (ASPCAP) by comparing its output to the results from several literature studies. In Section 3 we explain the data collection for our KOI sample. In Section 4 we present our analysis of the KOI planet and host star parameters, focusing on orbital period and $[\mathrm{Fe} / \mathrm{H}]$, and in Sections 5 and 6 we discuss the interpretation of our results and final conclusions, respectively.

\section{Validating APOGEE Spectroscopic Parameters}

All the data in this work were collected as part of APOGEE in the 14th Data Release (DR14, Abolfathi et al. 2017) of the third and fourth Sloan Digital Sky Survey (Eisenstein et al. 2011; Blanton et al. 2017). APOGEE utilizes a multiobject spectrograph (Wilson et al. 2010, 2012) mounted on the Sloan $2.5 \mathrm{~m}$ telescope (Gunn et al. 2006) to sample up to 300 sources simultaneously with high-resolution $(R \sim 22,500)$, high signal-to-noise ratio $(\mathrm{S} / \mathrm{N}>100), H$-band $(1.5-1.7 \mu \mathrm{m})$ spectroscopy. Details on the motivation and scope of the APOGEE survey are described in Majewski et al. (2017), and the targeting is described in Zasowski et al. (2013). All of the data from APOGEE are processed through automated reduction and stellar parameter pipelines (Holtzman et al. 2015; Nidever et al. 2015), and the spectroscopic parameters used for the stars in our sample are derived from the Automated Stellar Parameters and Chemical Abundances Pipeline (ASPCAP). We give a brief overview of ASPCAP here for convenience, 
Table 1

Spectral Synthesis Study Comparison Parameters

\begin{tabular}{|c|c|c|c|c|c|c|c|c|c|c|}
\hline \multirow{2}{*}{ APOGEE ID } & \multicolumn{2}{|c|}{ ASPCAP } & \multicolumn{2}{|c|}{ Bruntt et al. (2012) } & \multicolumn{2}{|c|}{$\begin{array}{l}\text { Buchhave } \\
\text { et al. (2012) }\end{array}$} & \multicolumn{2}{|c|}{ Huber et al. (2013) } & \multicolumn{2}{|c|}{ Brewer et al. (2016) } \\
\hline & $T_{\text {eff }}(\mathrm{K})$ & {$[\mathrm{Fe} / \mathrm{H}]$} & $T_{\text {eff }}(\mathrm{K})$ & {$[\mathrm{Fe} / \mathrm{H}]$} & $T_{\mathrm{eff}}(\mathrm{K})$ & {$[\mathrm{m} / \mathrm{H}]$} & $T_{\mathrm{eff}}(\mathrm{K})$ & {$[\mathrm{Fe} / \mathrm{H}]$} & $T_{\text {eff }}(\mathrm{K})$ & {$[\mathrm{Fe} / \mathrm{H}]$} \\
\hline $2 \mathrm{M} 19452396+4404359$ & 6425 & 0.04 & 6344 & 0.01 & $\ldots$ & $\ldots$ & $\ldots$ & $\cdots$ & $\ldots$ & $\cdots$ \\
\hline $2 \mathrm{M} 19455565+4400329$ & 4882 & 0.08 & $\cdots$ & $\cdots$ & 5082 & 0.03 & 5009 & -0.02 & $\cdots$ & $\cdots$ \\
\hline $2 \mathrm{M} 19480452+5024323$ & 5927 & 0.10 & $\cdots$ & $\cdots$ & 6040 & 0.11 & $\cdots$ & $\cdots$ & 6008 & 0.18 \\
\hline $2 \mathrm{M} 19542140+4045024$ & 6096 & -0.03 & $\cdots$ & $\cdots$ & $\ldots$ & $\ldots$ & 6081 & -0.03 & $\cdots$ & $\cdots$ \\
\hline $2 \mathrm{M} 20015142+4421140$ & 6070 & -0.33 & 6114 & -0.36 & $\ldots$ & $\ldots$ & $\ldots$ & $\cdots$ & $\cdots$ & $\cdots$ \\
\hline
\end{tabular}

(This table is available in its entirety in machine-readable form.)

but for details on the pipeline we refer the reader to García Pérez et al. (2016).

ASPCAP consists of two principal components: a FORTRAN90 optimization code (FERRE; Allende Prieto et al. $2006^{15}$ ) that compares the observed APOGEE spectra to synthetic libraries, and a multifunctional IDL wrapper used for bookkeeping and reading and preparing the input APOGEE spectra. FERRE performs a $\chi^{2}$ minimization to find the best-fit set of atmospheric parameters (effective temperature, $T_{\text {eff }}$; surface gravity, $\log g$; microturbulent velocity, $\xi_{t}$; and general solar-scaled metallicity, $[\mathrm{M} / \mathrm{H}]$ ), as well as $\mathrm{C}, \mathrm{N}$, and $\alpha$-element abundances from an interpolated library of synthetic ATLAS9 or MARCS model atmospheres. The atomic and molecular line list, gathered from the literature, has been updated regularly and is described most recently in Shetrone et al. (2015) and J. Holtzman et al. (2017, in preparation).

Once fundamental atmospheric parameters are found, ASPCAP extracts individual chemical abundances by fitting spectral windows optimized for each element. Iron has dozens of Fe I lines in the $H$ band, and Fe abundances are computed using $~ 55$ spectral windows. ASPCAP provides both raw and calibrated values for all of its spectroscopic parameters. Calibrated $T_{\text {eff }}$ values are established using observations of globular and open clusters, and by requiring that there are no trends of abundances with $T_{\text {eff }}$ in clusters (J. Holtzman et al. 2017, in preparation). In this study, we adopt the DR14 calibrated $T_{\text {eff }}$ and metallicity $([\mathrm{Fe} / \mathrm{H}])$ values.

Because ASPCAP is optimized for red giants and not well tested for dwarfs (the topic of a separate publication in preparation), it is worthwhile to test the pipeline's performance against published spectroscopic studies of dwarfs. We select comparison studies focused on planet search targets, because the stellar samples are similar to those in our study (i.e., consisting mostly of FGK dwarfs). We compare first to four large surveys that derive stellar parameters using spectral synthesis (Bruntt et al. 2012; Buchhave et al. 2012; Huber et al. 2013; Brewer et al. 2016), each with enough stars to give a substantive comparison of ASPCAP's performance. These data and comparisons are shown in Table 1 and Figure 1, top panels. We also compare ASPCAP's performance to equivalent width (EW) analysis studies of detailed chemical abundances (Ghezzi et al. 2010; Adibekyan et al. 2012; Nissen et al. 2014; Schuler et al. 2015) to better gauge the pipeline's performance. These data and comparisons are shown in Table 2 and Figure 1, bottom panels. The EW studies employ different methodologies, which allows us to compare ASPCAP against multiple strategies for deriving spectroscopic parameters. Summaries of

\footnotetext{
$\overline{15}$ Available from github.com/callendeprieto/ferre.
}

these comparisons with ASPCAP's performance are given in Sections 2.2 and 2.3.

\subsection{ASPCAP Internal Errors on $[\mathrm{Fe} / \mathrm{H}]$}

Each of the comparisons described below represents an estimate of the relative accuracy of the ASPCAP stellar parameter results, but not their precision. To first assess the internal error on the ASPCAP $[\mathrm{Fe} / \mathrm{H}]$ values, we turn to the sample of stars in the solar-age open cluster M67 that was observed by APOGEE, with parameters derived in the same way as our sample of KOIs. From the astrometric survey of Yadav et al. (2008), we selected the stars that were observed by APOGEE having the highest M67 membership probability $(\geqslant 90 \%)$ and also had ASPCAP uncalibrated $\log g$ values ${ }^{16}$ $\geqslant 4.0$ and measured $[\mathrm{Fe} / \mathrm{H}]$ values. From this sample of 76 stars, the $[\mathrm{Fe} / \mathrm{H}]$ median is $-0.021 \mathrm{dex}$, the mean is $-0.018 \mathrm{dex}$, and the standard deviation is 0.073 dex. However, as described below (Section 4.1), in our analysis we include only high-S/N (>100) spectra and exclude stars with $T_{\text {eff }}<4000 \mathrm{~K}$; performing the same cut to the M67 sample results in 46 stars with an $[\mathrm{Fe} / \mathrm{H}]$ median of $-0.016 \mathrm{dex}$, a mean of $-0.011 \mathrm{dex}$, and a standard deviation of 0.053 dex.

M67 is known to be chemically inhomogeneous-Liu et al. (2016) found a metallicity difference of $\sim 0.05$ dex between a solar twin and a solar analog in M67, as well an enhancement of $\sim 0.05$ dex in neutron-capture elements in the solar analog versus the solar twin. Additionally, using SDSS-III DR12 APOGEE data, Bertran de Lis et al. (2016) found the spread in $[\mathrm{O} / \mathrm{Fe}]$ in cool, low-gravity stars $\left(4000<T_{\text {eff }}<4600 \mathrm{~K}, \log g<\right.$ $3.8)$ in M67 to be higher $\left(\sigma[\mathrm{O} / \mathrm{Fe}]_{\mathrm{err}} \sim 0.03 \mathrm{dex}\right)$ as compared to other solar- or supersolar-metallicity clusters NGC 6791 and NGC 6819, which show $\sigma[\mathrm{O} / \mathrm{Fe}]_{\text {err }} \lesssim 0.01$ dex. However, only a handful of dwarf stars in NGC 6791 and NGC 6819 were targeted by APOGEE, and even fewer make our S/N cut. Thus, we adopt the $\sigma[\mathrm{Fe} / \mathrm{H}]$ value from M67, $0.053 \mathrm{dex}$, as a conservative error (since a significant part of the spread in $[\mathrm{Fe} / \mathrm{H}]$ is likely intrinsic to the cluster) on the ASPCAP metallicities for the KOIs in our analysis henceforth.

\subsection{Comparison to Literature-Spectral Synthesis Studies}

Bruntt et al. (2012) utilized the analysis package VWA (Bruntt et al. 2010) to derive stellar parameters and elemental abundances for a sample of $93 \mathrm{G}$ dwarfs in the Kepler field. Their data consist of high-resolution $(R \approx 80,000)$ and high-S/N $(\sim 200-300)$

\footnotetext{
16 The spectroscopic surface gravities for dwarfs in APOGEE DR14 are significantly lower than what is expected from stellar isochrones, and an acceptable calibration relation has not yet been developed by the ASPCAP team.
} 

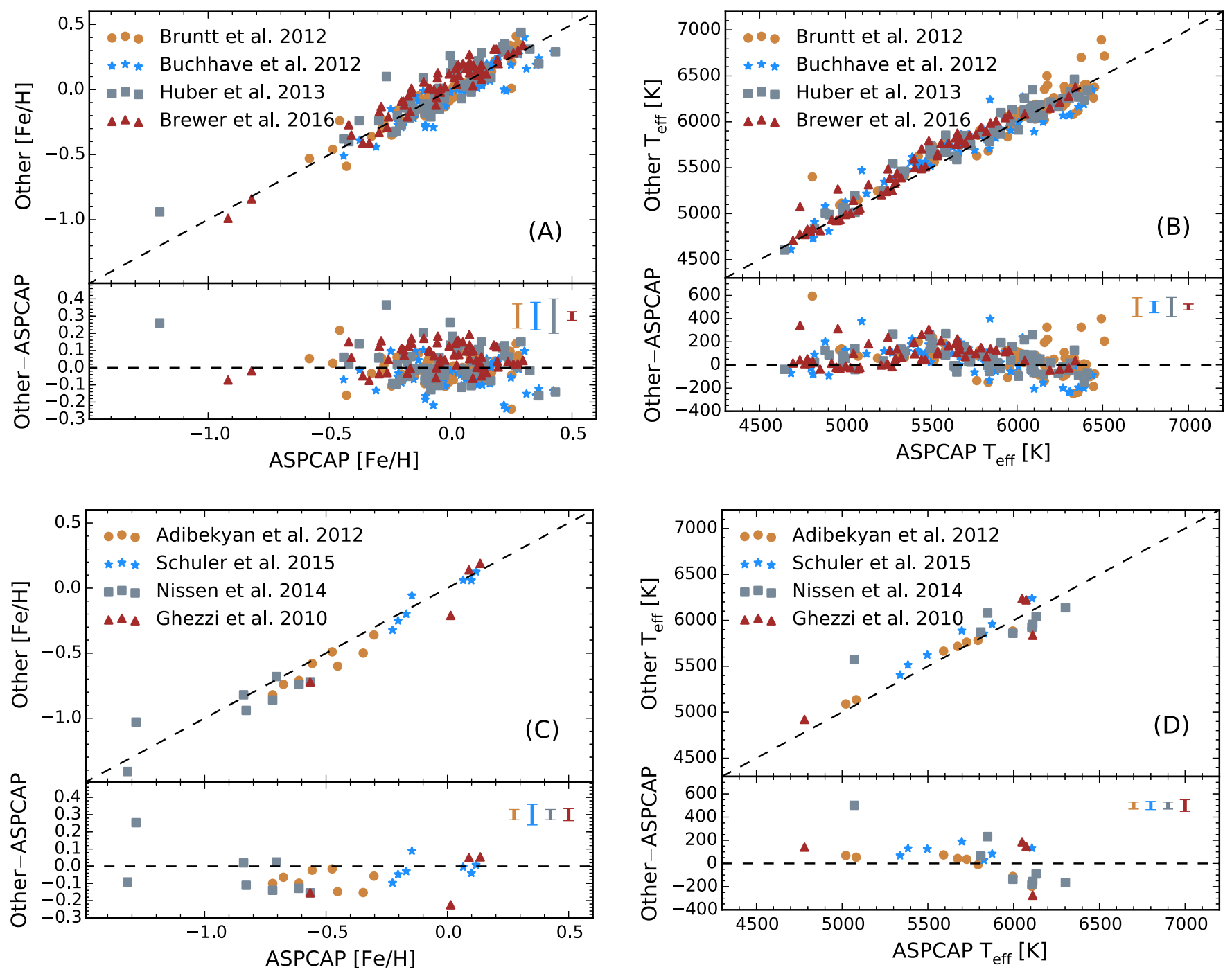

Figure 1. Comparisons of APOGEE $[\mathrm{Fe} / \mathrm{H}]$ and $T_{\text {eff }}$ measurements to values determined in the literature. Each plot shows the $T_{\text {eff }}$ and [Fe/H] values from ASPCAP and the literature plotted against each other, as well as the difference (Other-ASPCAP). For the top panels, the dotted black line shows the one-to-one relationship, and in the bottom panels, the dotted black line shows the line of no difference. Typical errors from the literature studies are shown in the top right of each bottom panel. (a) ASPCAP's [Fe/H] determinations compared to measurements from synthesis studies. As a whole, the difference (Other-ASPCAP) shows good agreement with an rms scatter of 0.09 dex and a mean offset of 0.00 dex. (b) APOGEE $T_{\text {eff }}$ measurements compared to those from synthesis studies. Though there is a slight bump around $T_{\text {eff }} \sim 5500 \mathrm{~K}$, APOGEE shows excellent overall agreement with these surveys, with an rms scatter of $129 \mathrm{~K}$ and mean offset of $57 \mathrm{~K}$. (c) APOGEE's [Fe/H] determination compared to equivalent width studies in the literature. The difference in measured iron abundances by APOGEE and these studies show a mean difference of -0.05 dex and an rms scatter of 0.11 dex. (d) APOGEE's $T_{\text {eff }}$ determination compared to equivalent width studies in the literature. The difference in measured temperatures by APOGEE and these dedicated studies show a mean offset of $36 \mathrm{~K}$ and an rms scatter of $166 \mathrm{~K}$.

optical spectra. The accuracy of their parameters resulted from adopting asteroseismic $\log g$ values, which they held fixed to derive the rest of their parameters. Bruntt et al. (2012) reported typical uncertainties in their $T_{\text {eff }}$ and $[\mathrm{Fe} / \mathrm{H}]$ determinations of $80 \mathrm{~K}$ and $0.07 \mathrm{dex}$, respectively. The overlapping sample with APOGEE contains 71 stars. The differences (ASPCAP-Other) in the effective temperature $\left(\Delta T_{\text {eff }}\right)$ and iron abundance $(\Delta[\mathrm{Fe} / \mathrm{H}])$ determinations between ASPCAP and Bruntt et al. (2012) have mean offsets and rms scatter of $48 \pm 147 \mathrm{~K}$ and $0.00 \pm 0.07$ dex.

Buchhave et al. (2012) systematically derived spectroscopic and stellar parameters for a sample of 152 planet-hosting stars (PHSs) with 1500 observations from multiple telescopes and spectrographs, having $\mathrm{S} / \mathrm{N} \geqslant 30$. The final stellar parameters were determined from the average of all the measurements of each particular star. For this study, the authors developed their own analysis package, SPC, designed to analyze spectra with low to modest $\mathrm{S} / \mathrm{N}$. The authors claimed typical uncertainties in the $T_{\text {eff }}$ and $[\mathrm{Fe} / \mathrm{H}]$ of $50 \mathrm{~K}$ and 0.08 dex, respectively, which were derived as the scatter among their sample of measurements for each star. The overlap between Buchhave et al. (2012) and APOGEE is 75 stars. From these, the mean offset and rms scatter for $\Delta T_{\text {eff }}$ and $\Delta[\mathrm{Fe} / \mathrm{H}]$ are $43 \pm 147 \mathrm{~K}$ and $-0.04 \pm 0.09$ dex, respectively.

Huber et al. (2013) produced a catalog of fundamental stellar parameters for 66 PHSs in the Kepler field. Huber et al. (2013) used a combination of SME (Valenti \& Piskunov 1996) and SPC to obtain initial guesses of the $T_{\text {eff }},[\mathrm{Fe} / \mathrm{H}]$, and $\log g$. Stellar $\log g$ values were then fixed to asteroseismic solutions (derived using the initial $T_{\text {eff }}$ and $[\mathrm{Fe} / \mathrm{H}]$ from SPC and SME), and Huber et al. (2013) re-derived $T_{\text {eff }}$ and $[\mathrm{Fe} / \mathrm{H}]$ with the asteroseismic constraints in place. As with those from Buchhave et al. (2012), the Huber et al. (2013) data come from multiple telescopes, have modest $\mathrm{S} / \mathrm{N}$, and were analyzed in the same way for their 
Table 2

Equivalent Width Study Comparison Parameters

\begin{tabular}{|c|c|c|c|c|c|c|c|c|c|c|}
\hline \multirow{2}{*}{ APOGEE ID } & \multicolumn{2}{|c|}{ ASPCAP } & \multicolumn{2}{|c|}{$\begin{array}{l}\text { Adibekyan } \\
\text { et al. (2012) }\end{array}$} & \multicolumn{2}{|c|}{$\underline{\text { Schuler et al. (2015) }}$} & \multicolumn{2}{|c|}{ Nissen et al. (2014) } & \multicolumn{2}{|c|}{ Ghezzi et al. (2010) } \\
\hline & $\overline{T_{\text {eff }}(\mathrm{K})}$ & {$[\mathrm{Fe} / \mathrm{H}]$} & $\overline{T_{\text {eff }}(\mathrm{K})}$ & {$[\mathrm{Fe} / \mathrm{H}]$} & $\overline{T_{\mathrm{eff}}(\mathrm{K})}$ & {$[\mathrm{Fe} / \mathrm{H}]$} & $T_{\text {eff }}(\mathrm{K})$ & {$[\mathrm{Fe} / \mathrm{H}]$} & $T_{\text {eff }}(\mathrm{K})$ & {$[\mathrm{Fe} / \mathrm{H}]$} \\
\hline $2 \mathrm{M} 02360498+0653140$ & 4781 & 0.01 & $\ldots$ & $\ldots$ & $\ldots$ & $\ldots$ & $\ldots$ & $\ldots$ & 4922 & -0.21 \\
\hline $2 \mathrm{M} 02515835+1122119$ & 5809 & -0.70 & $\ldots$ & $\ldots$ & $\ldots$ & $\ldots$ & 5873 & -0.68 & $\ldots$ & $\ldots$ \\
\hline 2M03402202-0313005 & 5996 & -0.72 & 5884 & -0.82 & $\cdots$ & $\ldots$ & 5859 & -0.86 & $\cdots$ & $\ldots$ \\
\hline $2 \mathrm{M} 13121982+1731016$ & 6112 & -0.57 & $\cdots$ & $\cdots$ & $\cdots$ & $\cdots$ & 5956 & -0.72 & 5837 & -0.72 \\
\hline $2 \mathrm{M} 19134816+4014431$ & 5875 & -0.17 & $\cdots$ & $\cdots$ & 5958 & -0.20 & $\cdots$ & $\cdots$ & $\cdots$ & $\cdots$ \\
\hline
\end{tabular}

(This table is available in its entirety in machine-readable form.)

initial $T_{\text {eff }}$ and $[\mathrm{Fe} / \mathrm{H}]$ measurements. The authors reported an average uncertainty of $82 \mathrm{~K}$ and $0.1 \mathrm{dex}$ for $T_{\text {eff }}$ and $[\mathrm{Fe} / \mathrm{H}]$, respectively. All 66 stars in Huber et al. (2013) were observed with APOGEE. The mean offset and scatter for $\Delta T_{\text {eff }}$ and $\Delta[\mathrm{Fe} / \mathrm{H}]$ are $52 \pm 105 \mathrm{~K}$ and $0.02 \pm 0.10$ dex, respectively.

Brewer et al. (2016) used SME (Valenti \& Piskunov 1996) to provide a catalog of spectral and stellar properties for 1615 FGK dwarfs. All observations were taken with the HIRES spectrograph (Vogt et al. 1994) at the Keck I telescope with resolution $R \approx 70,000$, but they vary in $\mathrm{S} / \mathrm{N}$ (about $25 \%$ of their stars have $\mathrm{S} / \mathrm{N}<100$, and the rest have $\mathrm{S} / \mathrm{N} \geqslant 100$ ). Brewer et al. (2016) note a strong dependence of their derived parameters on $\mathrm{S} / \mathrm{N}$. Of the Brewer et al. (2016) subsample that was also observed with APOGEE, only five stars have $\mathrm{S} / \mathrm{N}<100$. These authors reported a typical uncertainty in $T_{\text {eff }}$ of $\sim 25 \mathrm{~K}$ and a typical $[\mathrm{Fe} / \mathrm{H}]$ uncertainty of 0.01-0.04 dex. Of the 1615 stars in their sample, 60 have also been observed by APOGEE. In this overlapping sample, we find a mean offset and scatter for $\Delta T_{\text {eff }}$ and $\Delta[\mathrm{Fe} / \mathrm{H}]$ of $82 \pm 126 \mathrm{~K}$ and $0.06 \pm 0.10 \mathrm{dex}$, respectively.

\subsection{Comparison to Literature-EW Studies}

In addition to the large surveys described above, we compare ASPCAP's results with a few select studies that have computed stellar parameters and derived elemental abundances through EW measurements. Specifically, we compare ASPCAP's results with Ghezzi et al. (2010), Adibekyan et al. (2012), Nissen et al. (2014), and Schuler et al. (2015). In all of the following studies, stellar parameters were determined by adjusting the parameters until there was no correlation between the $[\mathrm{Fe} / \mathrm{H}]$ values derived from $\mathrm{Fe}$ I lines and the lower excitation potential $(\chi)$ of the lines, nor between $[\mathrm{Fe} \mathrm{I} / \mathrm{H}]$ and reduced $\mathrm{EW}[\log (\mathrm{EW} / \lambda)]$, and until there was agreement between abundances derived from Fe I and Fe II lines.

Ghezzi et al. (2010) obtained spectra of a sample of 117 PHSs from the Fiber-fed Extended Range Optical Spectrograph (FEROS; Kaufer et al. 1999). The setup chosen by Ghezzi et al. (2010) resulted in spectral coverage from 3560 to $9200 \AA$ and a nominal resolution of $R \approx 48,000$. The reported typical $\mathrm{S} / \mathrm{N}$ per resolution element ranges from $\sim 200$ to 500 . EWs were measured using the code ARES (Sousa et al. 2007), and stellar parameters were derived using the 2002 version of MOOG (Sneden 1973) assuming local thermodynamic equilibrium (LTE). The uncertainties for this sample were derived with the method outlined in Gonzalez (1998). Ghezzi et al. (2010) report typical $T_{\text {eff }}$ uncertainties ranging from $\sim 30$ to $70 \mathrm{~K}$ and typical $[\mathrm{Fe} / \mathrm{H}]$ uncertainties ranging from $\sim 0.02$ to 0.05 dex. For the four stars observed by APOGEE and Ghezzi et al. (2010), the mean offset and rms scatter in $\Delta T_{\text {eff }}$ between ASPCAP and
Ghezzi et al. are $51 \pm 195 \mathrm{~K}$, and the mean offset and scatter in $\Delta[\mathrm{Fe} / \mathrm{H}]$ are $0.07 \pm 0.14 \mathrm{dex}$, indicating a fair agreement.

Adibekyan et al. (2012) obtained a sample of 1111 FGK dwarfs from the HARPS (High Accuracy Radial velocity Planet Searcher) GTO (Guaranteed Time Observations) planet search program (Mayor et al. 2003; Lo Curto et al. 2010; Santos et al. 2011). The spectra taken from the HARPS spectrograph (Mayor et al. 2003) have spectral resolution $R \approx 110,000$ and an $\mathrm{S} / \mathrm{N}$ ranging from $\sim 20$ to 2000 , where $84 \%$ of the sample has $S / N \geqslant 100$. Their sample consists of dwarfs similar in $T_{\text {eff }}$ to the Sun, the majority of which lie within 4500-6500 K and with metallicities ranging from -1.39 to 0.55 dex. The analysis was completed in a similar manner to Ghezzi et al. (2010), by assuming LTE, generating a grid of Kurucz model atmospheres (Kurucz 1993), and making use of ARES and MOOG. However, because Ghezzi et al. limited their line list to lines with $\log g f$ values measured in the lab, the analysis of Adibekyan et al. (2012) benefits from a more extensive line list. The uncertainties from Adibekyan et al. (2012) were determined by adding quadratically the uncertainties in the parameters of the atmospheric model and the scatter measured among the abundances of each individual line. Since HARPS is in the southern hemisphere, there are only eight stars that were also observed with APOGEE. The reported uncertainties were typically about $30 \mathrm{~K}$ for $T_{\text {eff }}$ and $0.03 \mathrm{dex}$ in $[\mathrm{Fe} / \mathrm{H}]$. The mean offset and rms scatter of $\Delta T_{\text {eff }}$ and $\Delta[\mathrm{Fe} / \mathrm{H}]$ with respect to ASPCAP are $-6 \pm 92 \mathrm{~K}$ and $-0.08 \pm$ 0.09 dex, respectively.

Nissen et al. (2014) measured the $\mathrm{C} / \mathrm{O}$ ratio in a sample of 66 Sun-like stars, with $T_{\text {eff }}$ ranging from 5400 to $6400 \mathrm{~K}$. The Nissen et al. (2014) data are from both HARPS and FEROS, with the same configurations as in Adibekyan et al. (2012) and Ghezzi et al. (2010). The sample of stars observed with HARPS has $\mathrm{S} / \mathrm{N} \gtrsim 300$, while the FEROS-observed stars have a typical $\mathrm{S} / \mathrm{N} \sim 200$. Nissen et al. (2014) derive $T_{\text {eff }}$ in their HARPS-FEROS sample using photometric calibrations and initially derive $[\mathrm{Fe} / \mathrm{H}]$ values by interpolating within the planeparallel model atmosphere MARCS grid (Gustafsson et al. 2008). They then derive more accurate $[\mathrm{Fe} / \mathrm{H}]$ values by measuring the EWs of Fe II lines using the Uppsala program EQWIDTH. Nissen et al. report a typical uncertainty in $[\mathrm{Fe} / \mathrm{H}]$ of $0.03 \mathrm{dex}$ and internal, $1 \sigma T_{\text {eff }}$ errors of $30 \mathrm{~K}$. Though Nissen et al. do not explicitly describe how they derive their errors, they state that their errors are drawn from uncertainties in their model atmosphere fits and EW measurements. Comparing the eight stars in Nissen et al. that were also observed by APOGEE, the mean offset and rms scatter of $\Delta T_{\text {eff }}$ and $\Delta[\mathrm{Fe} / \mathrm{H}]$ are $8 \pm 230 \mathrm{~K}$ and $-0.04 \pm 0.14$ dex, respectively. 
Schuler et al. (2015) derived stellar parameters and elemental abundances for seven PHSs identified by Kepler. The data were collected from HIRES on the Keck I telescope as part of the Kepler Follow-up Observing Program (KFOP; Gautier et al. 2007). The KFOP spectra have a spectral coverage of $3650-7950 \AA$ and a spectral resolution of $R \approx 50,000$. Schuler et al. only considered data with $S / N \geqslant 150$. Stellar parameters and abundances for this study were extracted using an LTE, curve-of-growth analysis. EWs were measured using the analysis package SPECTRE, and abundances were derived from the 2014 version of MOOG, along with synthetic fits to the data interpolated from the ATLAS9 Kurucz model atmosphere grid. Uncertainties in $T_{\text {eff }}$ are reported as the difference between the adopted $T_{\text {eff }}$ value and the value that results in a $1 \sigma$ correlation between the $[\mathrm{Fe} / \mathrm{H}]$ versus $\chi$ and reduced $\mathrm{EW}$ relations. Schuler et al. report uncertainties in $T_{\text {eff }}$ between 25 and $45 \mathrm{~K}$ and uncertainties in $[\mathrm{Fe} / \mathrm{H}]$ between 0.04 and 0.08 dex. In the Schuler et al. sample, all seven stars in the Schuler et al. sample were also observed with APOGEE, resulting in a mean offset and scatter between the two studies for $\Delta T_{\text {eff }}$ and $\Delta[\mathrm{Fe} / \mathrm{H}]$ of $110 \pm 119 \mathrm{~K}$ and $-0.02 \pm 0.06 \mathrm{dex}$, respectively.

Overall, after these various comparisons with multiple studies, we find that ASPCAP is accurate for $T_{\text {eff }}$ and $[\mathrm{Fe} / \mathrm{H}]$ within the scatter and agree with these multiple optical studies that utilize different methodologies. We note that the mean offset in $T_{\text {eff }}(57 \mathrm{~K})$ versus the synthesis studies indicates that ASPCAP may be underreporting the $T_{\text {eff }}$ compared to these other studies. However, we find almost no offsets in $T_{\text {eff }}$ as compared to the studies of Nissen et al. (2014) and Adibekyan et al. (2012). Furthermore, because $-57 \mathrm{~K}$ is well within the rms scatter for each of these studies, we do not consider it to be problematic for our purposes. Taking all these comparison studies into account, we find that the mean offset and rms scatter in $\Delta[\mathrm{Fe} / \mathrm{H}](0.004$ and 0.10 dex, respectively) are within the uncertainties required for this work.

Having validated the performance of ASPCAP, we now move on to the study of a particular subset of APOGEE data consisting of repeated observations of KOIs resulting in high$\mathrm{S} / \mathrm{N}$ spectra.

\section{APOGEE KOI RV Sample}

The primary goal of APOGEE, now in its second phase (APOGEE-2; Majewski et al. 2016), is to study the Milky Way through the RVs and chemical abundances of as many as half a million stars, chosen to be primarily red giants across multiple stellar populations and Galactic regions. Additional science programs are also included in the survey, with one such program monitoring KOIs to search for false positives through RV variations (Fleming et al. 2015). APOGEE data reach an $\mathrm{RV}$ precision of $\sim 100 \mathrm{~m} \mathrm{~s}^{-1}$ (Troup et al. 2016), allowing the search for eclipsing binaries (EBs) and other grazing incidence geometries that may resemble transiting planets in the initial Kepler reduction pipeline. The APOGEE survey will eventually observe $\sim 1050$ KOIs with $\geqslant 18$ epochs across five APOGEE-2 fields (roughly the size of Kepler tiles), with each KOI having a sufficient $\mathrm{S} / \mathrm{N}$ to get quality $\mathrm{RVs}$ at each epoch. As a result, the final "RV-normalized," summed spectra over all epochs are of very high $\mathrm{S} / \mathrm{N}$ (typically a few hundred), which allows for the derivation of high-precision stellar parameters and elemental abundances for planet-hosting stars. The APOGEE targets were chosen with the goal of observing all possible "Confirmed" or "Candidate" KOIs with $H<14$ in those five Kepler tiles. Some KOIs were excluded from the sample on the basis of unphysical impact parameters and planet radii consistent with stellar values. Currently APOGEE has observed $\sim 600$ KOIs from the Q1-Q16 catalog (Mullally et al. 2015), orbiting $\sim 450$ PHSs, each with between 10 and 28 epochs at the time of this study.

Data concerning the orbital and planetary parameters for each KOI were gathered using the public NASA Exoplanet Archive, which provides the information in the form of interactive tables of confirmed and candidate planetary and stellar properties and includes a suite of integrated analysis tools (Akeson et al. 2017). Use of this archive allows us to exclude known false positives and ensures that we are using the most up-to-date KOI dispositions in the literature. The specific targets included in our analysis, vetted from the $\sim 600 \mathrm{KOIs}$ observed thus far by APOGEE-2, are described below (Section 4.1).

\section{KOI Stellar Metallicity and Planet Period Relation}

\subsection{Selected Sample}

All of the stars in our KOI sample were observed as part of the APOGEE KOI Goal Program (Fleming et al. 2015), as described above. Initially, that consists of $624 \mathrm{KOIs}$ and 450 PHSs. To ensure the quality of the data, we restrict our analysis sample using a series of APOGEE flags and other constraints. ${ }^{17}$ We first remove data with any of the STARFLAGS BAD_PIXELS, VERY_BRIGHT_NEIGHBOR, and LOW_SNR set. It is worth mentioning that a fraction of our sample falls on the highpersistence region of APOGEE's "blue chip." However, we decide to keep these data since persistence effects were shown to be minimized in DR14 (J. Holtzmann et al. 2017, in preparation). To exclude unreliable ASPCAP fits defined as values close to the edge of the model atmosphere grid, we also remove data with any of the following ASPCAPFLAGS: TEFF_BAD, LOGG_BAD, and METALS_BAD set. Because the focus of this study is $[\mathrm{Fe} / \mathrm{H}]$, we remove KOIs with the PARAMFLAGS GRIDEDGE_BAD, CALRANGE_BAD, OTHER_BAD, and PARAM_FIXED flags set, with respect to the $[\mathrm{Fe} / \mathrm{H}]$ parameter. In addition, we require that all of the summed APOGEE spectra in our sample have $\mathrm{S} / \mathrm{N}>50$. Because the ASPCAP line list currently does not include $\mathrm{FeH}$ lines, which are important for modeling the metallicities of $\mathrm{M}$ dwarfs, we exclude all stars with $T_{\text {eff }}<4000 \mathrm{~K}$ (for a detailed discussion see Souto et al. 2017).

In addition to the quality cuts described above, we also trim our sample based on the orbital periods and inferred planet radii of the KOIs. To ensure that we avoid regions of parameter space associated with low survey completeness, we only analyze KOIs with orbital periods, $P<100$ days. ${ }^{18}$ For multiple-planet systems, we only analyze the planet with the shortest orbital period. We also restrict our sample to KOIs with inferred radii for the planet candidates $R_{p}<20 R_{\oplus}$. We anticipate that any planet candidates having radii larger than this limit are likely to be EBs. We correct for false positives from our sample by removing eight known EBs identified in the literature. We identify eight more likely binaries from visual inspection of spectra for which ASPCAP reported high

\footnotetext{
17 Descriptions of the APOGEE flags can be found at http://www.sdss.org/ dr13/algorithms/bitmasks/\#APOGEE_TARGET1.

18 For an estimate of survey completeness as a function of planet radius and orbital period, see Burke et al. (2015).
} 
$v \sin i$ values. In these cases, the reported $v \sin i$ values were a result of the combined spectra from the primary and companion stars. To remove any more potential EBs from our sample, we also filter stars with high RV variability, which we define as the ratio of the scatter of the RV measurements to the error of the $\mathrm{RV}$ measurements, given by

$$
\frac{\text { VSCATTER }}{\text { VERR_MED }}>17 \text {, }
$$

where VERR_MED is the median RV measurement error from all visits and VSCATTER is the rms scatter of all the RV measurements. Because the RV measurement errors are often underreported in APOGEE (Troup et al. 2016), we choose the cutoff as the median value for the 16 known binaries in our sample, which is $\sim 17$. After these cuts, our final sample consists of 282 KOIs (all unique PHSs), listed in Table 3.

The planet hosts in our sample are all FGK dwarfs with effective temperatures ranging from 4000 to $6500 \mathrm{~K}$. The $[\mathrm{Fe} / \mathrm{H}]$ of our sample range from -0.6 to 0.4 dex. We note that this parameter space is well covered by our tests of ASPCAP using literature comparisons (Sections 2.2, 2.3). The spectral $\mathrm{S} / \mathrm{N}$ in our KOI sample from APOGEE have a wide range; the inner $68 \%$ ranges in $\mathrm{S} / \mathrm{N}$ from 70 to 280 , while the asymmetric distribution peaks at $\mathrm{S} / \mathrm{N} \sim 140$ with a tail to $\mathrm{S} / \mathrm{N} \gtrsim 500$.

\subsection{Analysis and Results}

As discussed in Section 1, a correlation between an exoplanet's orbital period and the metallicity of its host star could indicate that protoplanetary disks with higher solid surface density cause planets to migrate or form closer to their host stars (Adibekyan et al. 2013; Beaugé \& Nesvorný 2013; Mulders et al. 2016). Alternatively or in addition, such a correlation could mean that metal-rich disks spawn planet cores faster at larger orbital distances, facilitating the cores growing into gas-enveloped planets with larger $R_{p}$ and thus causing an absence of strictly rocky planets at longer periods around metal-rich stars (Dawson et al. 2015). Given the multiple interpretations and somewhat contradictory results regarding these host/planet properties (e.g., Winn et al. 2017), we want to assess the presence and strength of the $[\mathrm{Fe} / \mathrm{H}]-P$ correlation within the APOGEE KOI sample.

To assess first the correlation between host $[\mathrm{Fe} / \mathrm{H}]$ and orbital period, we perform two different nonparametric tests, calculating Kendall's rank correlation coefficient, $\tau$ (Kendall 1938), and Spearman's rank correlation coefficient, $\rho$ (Spearman 1904). Kendall's $\tau$ coefficient is $\tau=-0.21$, with a $p$-value $p_{\tau}=$ $1.40 \times 10^{-7}$ (the equivalent of a $5.1 \sigma$ deviation from a normal distribution). Spearman's rank correlation coefficient is $\rho=$ -0.31 with a $p$-value $p_{\rho}=1.67 \times 10^{-6}(5.1 \sigma)$. These results indicate that as the host star $[\mathrm{Fe} / \mathrm{H}]$ increases, the orbital periods of the planets around those stars decrease. To verify the robustness of these correlations, we perform a Monte Carlo simulation with $10^{5}$ sets of data. For every simulated data set, we add a perturbation that is randomly drawn from a normal distribution with our adopted $1 \sigma$ uncertainty of $0.053 \mathrm{dex}$ (see Section 2.1) to the ASCAP-derived host $[\mathrm{Fe} / \mathrm{H}]$ for each KOI. We then recalculate $\rho$ and $\tau$ for each simulated data set. For Kendall's rank correlation, we recover the significance of this trend at a $4.83_{-0.30}^{+0.31} \sigma$ level, and for Spearman's rank correlation we recover a significance of $4.83_{-0.31}^{+0.31} \sigma$, where the errors represent the inner $68 \%$ of the posterior distribution.

To analyze further the correlation between orbital period and host $[\mathrm{Fe} / \mathrm{H}]$, we adopt a method similar to that employed by Buchhave et al. (2014). We generate $10^{4}$ test orbital periods equally separated in log space, spanning from the minimum to the maximum planetary orbital period in our sample. For each of these periods, ${ }_{P i}$, we divide our KOI sample into two bins, one where the KOIs have orbital period $P>P_{i}$ and one with $P \leqslant P_{i}$. We then use a two-sample Kolmogorov-Smirnov (K-S) test, as well as a $k$-sample Anderson-Darling (A-D) test for redundancy, to determine the likelihood that the host star iron abundances from the "short"- versus "long"-period bins are drawn randomly from the same parent distribution. We find a critical period, $P_{\text {crit }}$, where this likelihood is minimized (see Figure 2). In the case where the minimum $p$ value is equal among more than one period, it is because we are oversampling our period distribution. In this case, we take the mean. Within our data set we find $P_{\text {crit }}=8.3$ days by the K-S test and the A-D test, with $p$ values of $p_{\mathrm{ks}}=5.0 \times 10^{-7}(4.9 \sigma)$ and $p_{\mathrm{ad}}=$ $8.6 \times 10^{-6}(4.3 \sigma)$. To test the robustness of this critical period, we perform a Monte Carlo analysis and simulate $10^{4}$ sets of data, resampling as we did above, using the typical $[\mathrm{Fe} / \mathrm{H}]$ uncertainty of $0.053 \mathrm{dex}$ and the $P$ uncertainties as reported by the NASA Exoplanet Archive, which have a median of $4 \times 10^{-5}$ days. We recover the significance with both the $\mathrm{K}-\mathrm{S}$ test and A-D test at the $4.5_{-0.4}^{+0.4} \sigma$ level. We find $P_{\text {crit }}=8.3_{-4.1}^{+0.1}$ days for both the K-S test and A-D test, which is consistent with our original findings. This method thus discovers two unique $[\mathrm{Fe} / \mathrm{H}]$ populations within our data set, one that is supersolar on average and contains planets orbiting closer to the host star, and one that is solar metallicity on average and contains planets that orbit farther from their host. That is, planets with orbital periods $P \leqslant 8.3$ days have statistically more metal-enriched hosts than planets with $P>8.3$ days. This is the main finding of this paper. We note that performing the same tests on host star $[\mathrm{Fe} / \mathrm{H}]$ and planetary semimajor axis $(a)$, instead of period, produces similar results, with $a_{\text {crit }}=0.07$ au.

In Figure 2, we also note two other, less significant dips at $P \sim 22$ days and at $P \sim 70$ days. The A-D test found the shorter-period dip at $P \sim 21$ days, and the K-S test found it at $P \sim 23$ days. For the longer-period dip, the minimum $p$-value found with the $\mathrm{K}-\mathrm{S}$ test is at $P=63$ days, while the minimum found with the A-D test is at $P=71$ days. Running a Monte Carlo analysis with $10^{4}$ data sets, similar to the above but restricting our analysis within the range 15 days $<P<$ 45 days, we find the period that minimizes the $p$-value to be $P=22.7_{-1.5}^{+0.4}$ days for both the A-D and K-S tests. However, the significance with which we recover this period is only $3.5_{-0.5}^{+0.5} \sigma$ for both the A-D and K-S tests. Because of the lowered significance, we are not comfortable claiming significance at this period within our study. However, there is theoretical motivation to support a critical period around 23 days (see Section 5.2), and it warrants further work. To test the third dip, we perform the same analysis restricting our test periods to $P>45$ days, but the results are inherently less trustworthy considering the uneven sample sizes of KOIs with $P<65$ days and $P>65$ days. The period that minimizes the $p$-value for the A-D and K-S tests in this longest-period range is $69_{-9}^{+4}$ days, with a significance of $2.8_{-0.4}^{+0.4} \sigma$ for each test. For 
Table 3

Parameters of Selected Sample

\begin{tabular}{|c|c|c|c|c|c|c|c|c|c|c|c|}
\hline \multirow{2}{*}{ KOI } & \multirow{2}{*}{ KIC } & \multirow{2}{*}{$\begin{array}{l}\text { Period } \\
\text { (days) }\end{array}$} & \multicolumn{2}{|c|}{ Period Error } & \multirow{2}{*}{$\begin{array}{l}\text { Planet Radius } \\
\qquad\left(R_{\oplus}\right)\end{array}$} & \multicolumn{2}{|c|}{ Planet Radius Error } & \multirow{2}{*}{$\begin{array}{c}K_{p} \\
(\mathrm{mag})\end{array}$} & \multirow{2}{*}{ APOGEE ID } & \multirow{2}{*}{$\begin{array}{l}T_{\text {eff }} \\
(\mathrm{K})\end{array}$} & \multirow{2}{*}{$\begin{array}{r}{[\mathrm{Fe} / \mathrm{H}]} \\
(\mathrm{dex}) \\
\end{array}$} \\
\hline & & & $\begin{array}{l}\text { Upper Bound } \\
\text { (days) }\end{array}$ & $\begin{array}{l}\text { Lower Bound } \\
\text { (days) }\end{array}$ & & $\begin{array}{l}\text { Upper Bound } \\
\quad\left(R_{\oplus}\right)\end{array}$ & $\begin{array}{c}\text { Lower Bound } \\
\quad\left(R_{\oplus}\right)\end{array}$ & & & & \\
\hline K00041.02 & 6521045 & 6.89 & $2.28 \mathrm{E}-05$ & $-2.28 \mathrm{E}-05$ & 1.30 & 0.08 & -0.07 & 11.20 & $2 \mathrm{M} 19253263+4159249$ & 5827 & 0.07 \\
\hline K00049.01 & 9527334 & 8.31 & 4.21E-05 & $-4.21 \mathrm{E}-05$ & 2.74 & 0.43 & -0.13 & 13.70 & $2 \mathrm{M} 19285977+4609535$ & 5831 & -0.07 \\
\hline K00084.01 & 2571238 & 9.29 & $3.83 \mathrm{E}-06$ & $-3.83 \mathrm{E}-06$ & 2.10 & 0.26 & -0.10 & 11.90 & $2 \mathrm{M} 19214099+3751064$ & 5437 & -0.02 \\
\hline K00100.01 & 4055765 & 9.97 & $1.22 \mathrm{E}-05$ & $-1.22 \mathrm{E}-05$ & 16.89 & 2.15 & -4.29 & 12.60 & $2 \mathrm{M} 19244270+3911581$ & 6336 & -0.32 \\
\hline K00103.01 & 2444412 & 14.91 & $1.28 \mathrm{E}-05$ & $-1.28 \mathrm{E}-05$ & 2.62 & 0.33 & -0.17 & 12.59 & $2 \mathrm{M} 19264400+3745057$ & 5485 & 0.06 \\
\hline K00119.01 & 9471974 & 49.18 & $2.48 \mathrm{E}-05$ & $-2.48 \mathrm{E}-05$ & 8.20 & 0.50 & -0.55 & 12.65 & $2 \mathrm{M} 19381420+4603443$ & 5584 & 0.33 \\
\hline K00156.02 & 10925104 & 5.19 & 8.92E-06 & $-8.92 \mathrm{E}-06$ & 1.0 & 0.08 & -0.07 & 13.74 & 2M19362914+4820582 & 4662 & 0.23 \\
\hline
\end{tabular}

(This table is available in its entirety in machine-readable form.) 

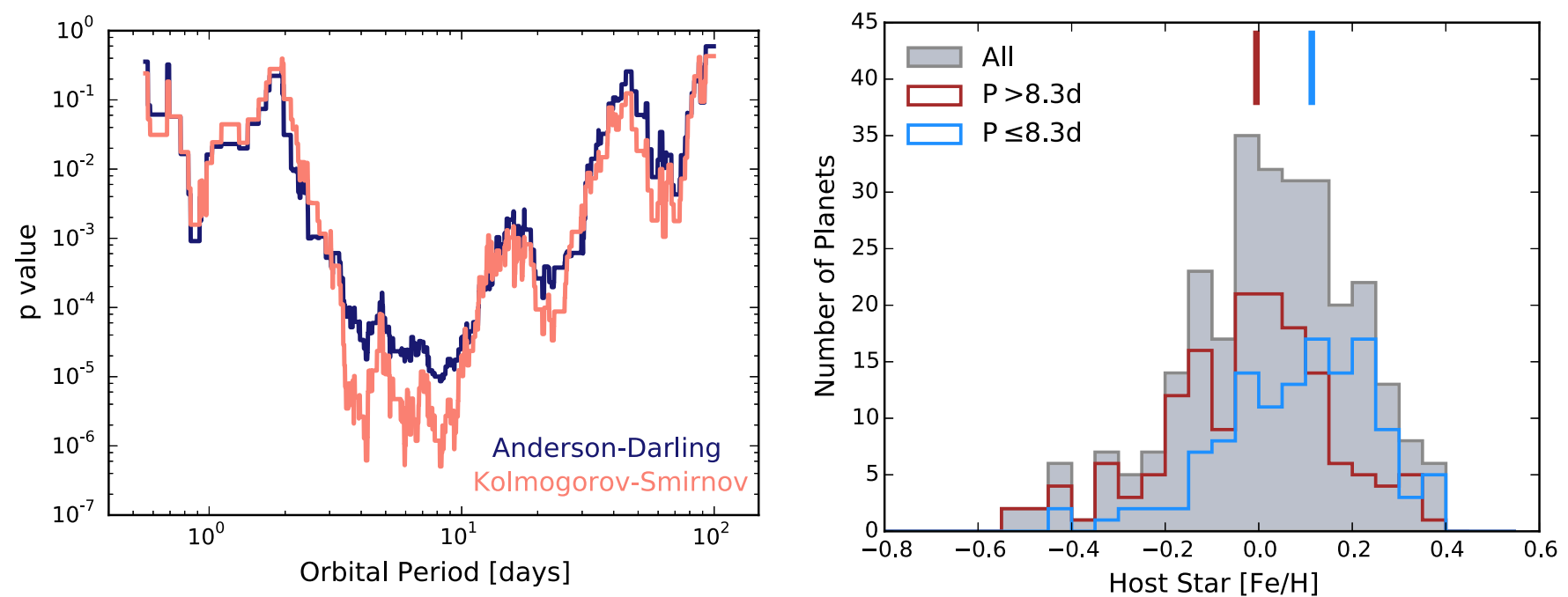

Figure 2. Left: $p$ values of the K-S and A-D tests for the probability that the $[\mathrm{Fe} / \mathrm{H}]$ distributions of exoplanet candidates above and below the given orbital period are drawn from the same parent distribution. There is a statistically significant dip at $P=8.2$ days in our sample, found by both an A-D test and a K-S test. Right: histogram of the host star metallicities of the long-period (red) and short-period (blue) populations, split by $P_{\text {crit }}=8.3$ days. The combined distribution is shown in gray. The long-period population peaks near solar metallicity, while the short-period population peaks above solar metallicity. The median host $[\mathrm{Fe} / \mathrm{H}]$ is shown by the tick marks for the long-period (red) and short-period (blue) populations.

the same reasons as the $P \sim 23$ day dip, we do not consider this a significant minimum, and currently we favor a twopopulation model split only at $P_{\text {crit }}=8.3_{-4.1}^{+0.1}$ days.

The iron abundance for the short-period population $\left(P \leqslant P_{\text {crit }}\right)$ is supersolar, with a median $[\mathrm{Fe} / \mathrm{H}]$ of $0.11 \mathrm{dex}$ and a standard deviation of $0.17 \mathrm{dex}$. The long-period population $\left(P>P_{\text {crit }}\right)$ is consistent with solar metallicity and has a standard deviation of 0.18 dex. To test whether the means of these two samples differ significantly, we perform a MannWhitney U-test (Mann \& Whitney 1947) between these two populations. The Mann-Whitney U-test gives the probability that two separate populations have the same underlying mean. The test returns a $p$ value of $p_{\mathrm{mw}}=2.28 \times 10^{-7}(5.0 \sigma)$ that the short- and long-period populations have the same mean metallicity. Thus, we can safely reject the null hypothesis that these distributions have the same mean, which is consistent with the results of the K-S and A-D tests that the two $[\mathrm{Fe} / \mathrm{H}]$ populations, separated by $P_{\text {crit }}$, are sampled from different parent populations.

To further analyze the significance of the correlation in our sample, we follow the analysis of Mulders et al. (2016) and use the Nadaraya-Watson estimator (Nadaraya 1964; Watson 1964) to calculate how the mean iron abundance varies with orbital period (see Figure 3). The kernel regression of the mean metallicity, $\overline{[\mathrm{Fe} / \mathrm{H}}_{\mathrm{KOI}}$, as a function of orbital period is given by

$$
\overline{[\mathrm{Fe} / \mathrm{H}]_{\mathrm{KOI}}}(P)=\frac{\sum_{i=0}^{n}[\mathrm{Fe} / \mathrm{H}]_{i} K\left(\log \left(P / P_{i}\right), \sigma\right)}{\sum_{i=0}^{n} K\left(\log \left(P / P_{i}\right), \sigma\right)},
$$

where $n$ is the number of exoplanet candidates in the sample, $[\mathrm{Fe} / \mathrm{H}]_{i}$ and ${ }_{P i}$ are the host star metallicity and orbital period of each exoplanet candidate, respectively, and we use a lognormal kernel with constant bandwidth, $\sigma$, given by

$$
K(\log x, \sigma)=\frac{1}{\sqrt{2 \pi} \sigma} e^{-0.5(\log x / \sigma)^{2}},
$$

where $x$ is an arbitrary, dimensionless variable. In line with Mulders et al. (2016), we adopt a bandwidth of $\sigma=0.29$.

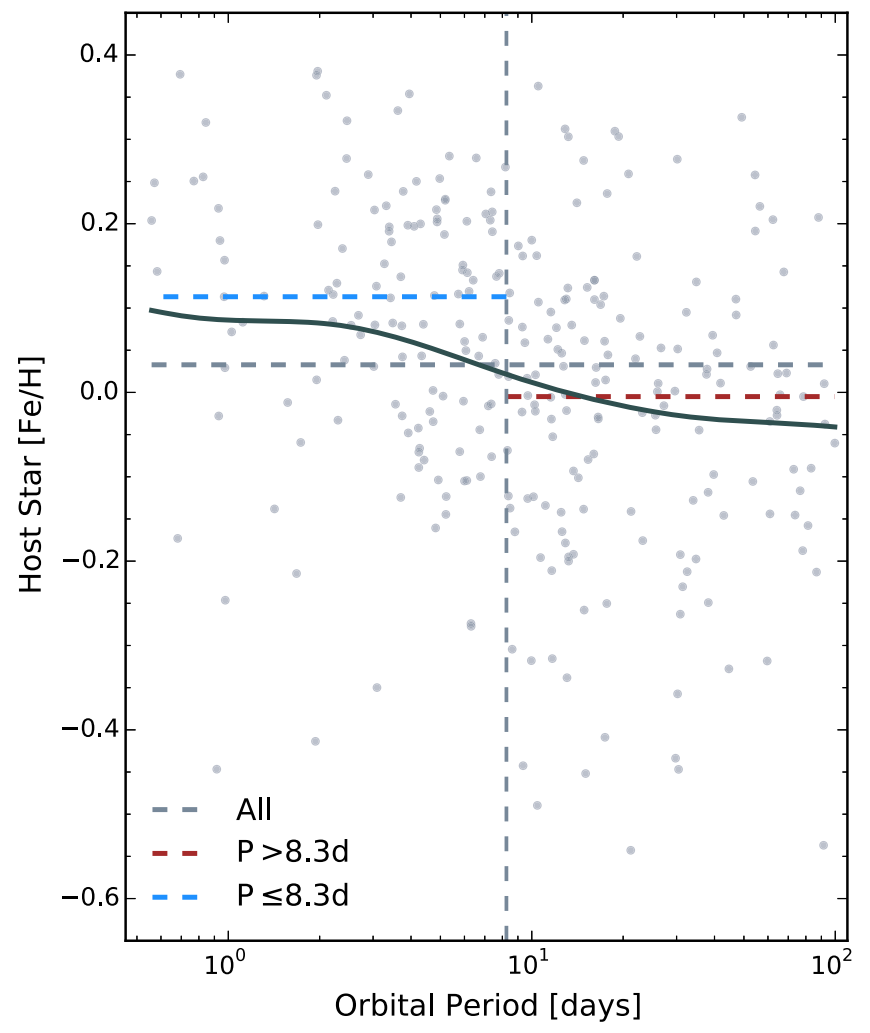

Figure 3. Host star metallicity as a function of exoplanet orbital period. The gray circles are the KOIs in our sample. The vertical dashed line is at $P=8.3$ days, which separates our short-period and long-period populations. The horizontal dashed lines show the median of the short-period (blue), long-period (red), and combined (gray) populations. The combined population has a median of $0.03 \mathrm{dex}$. The median metallicity of the short-period population is supersolar at $0.11 \mathrm{dex}$, while the median of the long-period population is 0.00 dex. The solid gray line is the kernel regression of the mean metallicity. The kernel regression shows a steady decrease from a maximum of $\sim 0.10$ dex at the shortest periods to a minimum of $\sim-0.04 \mathrm{dex}$ at the longest periods.

The kernel regression of the mean metallicity is plotted over all the KOIs in our sample in Figure 3, along with the median $[\mathrm{Fe} / \mathrm{H}]$ of the combined, short-period, and long-period samples. 
Using the kernel regression as a proxy for the mean $[\mathrm{Fe} / \mathrm{H}]$, we find that the maximum mean metallicity $([\mathrm{Fe} / \mathrm{H}] \sim 0.10$ dex $)$ occurs at the shortest period in our sample and the minimum mean metallicity in this trend $(\sim-0.04 \mathrm{dex})$ occurs at the longest period in our sample. This difference of $0.14 \mathrm{dex}$ is larger than the difference in the median metallicity between the long- and short-period samples by $\sim 0.03$ dex. However, the kernel regression clearly shows a decrease in mean $[\mathrm{Fe} / \mathrm{H}]$ in our sample at even the longest orbital periods.

\section{Discussion}

\subsection{Metallicity-Period Correlation}

Our results are consistent with those of Mulders et al. (2016), who find in their sample an increase of $0.15 \pm 0.05$ dex in host star metallicity for exoplanets orbiting at or interior to 10 days, as compared to longer-period planets. We find a statistically significant break in the KOI host star $[\mathrm{Fe} / \mathrm{H}]$-period distribution in our sample at 8.3 days, with shorter-period planets orbiting stars with a median $[\mathrm{Fe} / \mathrm{H}]$ of $0.11 \pm 0.17 \mathrm{dex}$ and longer-period planets orbiting stars with a median $[\mathrm{Fe} / \mathrm{H}]$ of $0.00 \pm 0.18$ dex. The Mann-Whitney and K-S probabilities in our data are $5.0 \sigma$ and $4.9 \sigma$, respectively, compared to Mulders et al. (2016), whose Mann-Whitney and K-S probabilities were comparable at $4.6 \sigma$ and $4.3 \sigma$, respectively. While our sample is significantly less than half the size (282 candidate or confirmed planets vs. Mulders's 665), our data are measured from higherresolution, high-S/N spectra, producing internal errors on $[\mathrm{Fe} / \mathrm{H}]$ of only $\sim 0.053$ dex and typical offsets from literature values of $\sim 0.00 \pm 0.09$ dex. The Mulders et al. (2016) study used LAMOST $[\mathrm{Fe} / \mathrm{H}]$ values, measured from $R \sim 2000$ optical spectra, typically with $\mathrm{S} / \mathrm{N} \leqslant 100$, and typical $[\mathrm{Fe} / \mathrm{H}]$ internal errors (evaluated by way of repeat observations of some stars) of $\sim 0.055$ dex and typical literatures offsets (using their calibrated $[\mathrm{Fe} / \mathrm{H}]$ values) of $\sim-0.06 \pm 0.18 \mathrm{dex}$. Thus, our study showcases an advantage APOGEE has over other surveys of similar scale. Even with our significantly smaller sample size compared to the Mulders et al. (2016) sample, we are able to recover the same correlation with greater confidence.

\subsubsection{Required Precision to Find the Trend}

Given our smaller sample size, what is the minimum $[\mathrm{Fe} / \mathrm{H}]$ precision required to be able to find the trend with orbital period that we do? To determine this precision, we replace the assumed $[\mathrm{Fe} / \mathrm{H}]$ error $(0.053 \mathrm{dex})$ with larger and larger errors until the resulting uncertainties on the significance of the K-S and A-D tests drop below $\sim 3 \sigma$. With our well-vetted (e.g., removal of stars showing signs of binarity in their RV variations) sample of high-resolution, high-S/N data, the greatest value that the mean $[\mathrm{Fe} / \mathrm{H}]$ error can take on is $\sim 0.1$ dex to still recover the observed trend with at least $3 \sigma$ significance.

\subsubsection{Possible Mechanisms}

What is the physical mechanism responsible for the observed trend between planetary orbital period and host star $[\mathrm{Fe} / \mathrm{H}]$ ? One possible explanation is that the dust sublimation radius (between 0.05 and 0.1 au, or $\sim 4-12$ days around a solar-mass star; Muzerolle et al. 2003; Eisner et al. 2005; Pinte et al. 2008; Min et al. 2011) in protoplanetary disks correlates with host star metallicity, and that it represents a semimajor axis cutoff inward of which no solids contribute to forming planets. If this were the case, we would expect a correlation between host star $T_{\text {eff }}$ (as the dust sublimation radius is known to depend on the stellar luminosity and/or stellar mass) and orbital period. To test this correlation, we performed Kendall's $\tau$ and Spearman's $\rho$ correlation tests among our KOI sample. Because we are testing a potential semimajor axis cutoff, we include only the closest planets in the case of multiple-planet systems, as we do with the rest of this study. We find $\tau=0.10$ with $p_{\tau}=0.05(1.7 \sigma)$ and $\rho=0.14$ with $p_{\rho}=0.06(1.6 \sigma)$, which indicates that there is no significant correlation between $T_{\text {eff }}$ and orbital period. To test further whether there is a difference in the host $T_{\text {eff }}$ among the short-period and long-period subsamples, we performed K-S and A-D tests, similar to the above analysis. For the K-S test we calculate $p_{\mathrm{ks}}=0.2(0.8 \sigma)$, and for the A-D test we calculate $p_{\mathrm{ad}}=0.07(1.3 \sigma)$. Thus, both of these tests indicate that there is no significant difference in the temperature distributions of the host stars in the short-period and long-period samples. The left panel of Figure 4 shows that while there is a slight decrease in $[\mathrm{Fe} / \mathrm{H}]$ from cooler to hotter $T_{\text {eff }}$, both the shorter-period/metal-rich and longer-period/ solar-metallicity subsamples decrease together with a roughly constant metallicity offset of $\sim 0.10$ dex. Because the correlation between $T_{\text {eff }}$ and $[\mathrm{Fe} / \mathrm{H}]$ is the same for both subsamples and the shorter-period and longer-period subsamples do not have significantly different temperature distributions (Figure 4, right panel), we do not believe that this decrease in $[\mathrm{Fe} / \mathrm{H}]$ at higher $T_{\text {eff }}$ affects our conclusions. Thus, our data rule out dust sublimation effects as a possible explanation for the metallicity-orbital period correlation. Mulders et al. (2015b) also ruled out the hypothesis of the dust sublimation radius controlling the semimajor axis cutoff in planetary occurrence rates, although these authors were testing a dependence of semimajor axis on stellar mass, not metallicity.

A second possible explanation for the observed trend between planetary orbital period and host star $[\mathrm{Fe} / \mathrm{H}]$ is that planets around higher-metallicity stars migrate inward and are "trapped" closer to their host stars (e.g., Kuchner \& Lecar 2002; Rein 2012; Plavchan \& Bilinski 2013). This closer location could be related to the dust sublimation radius-for which we do not see evidence as a factor in creating the $P-[\mathrm{Fe} / \mathrm{H}]$ trend as described above-or the co-rotation radius, where gas is accreted onto the stellar surface. Usually the co-rotation radius is within the dust sublimation radius (Najita et al. 2007, and references therein), and the former also depends on the angular velocity of the star, which does not have a strong stellar mass dependence during the pre-main-sequence stage (Bouvier 2013, and references therein). Thus, it is difficult to assess the likelihood of this "planet trapping" explanation within the context of this work. However, as discussed below, small, short-period planets around more metal-rich stars are generally rock dominated, indicating accretion and migration mainly within the snow line. Thus, their migration distance could not have been very far. The snow line is at a few au around a solarmass star after $\sim 1 \mathrm{Myr}$, but this distance for any given disk will depend on the stellar mass, as well as parameters like the heating mechanism(s), dust grain opacities, and the timing of planet formation (e.g., Kennedy \& Kenyon 2008; Martin \& Livio 2012; Mulders et al. 2015a; Xiao et al. 2017).

Finally, we consider the possibility that the correlation between planet orbital period and stellar metallicity could be a 

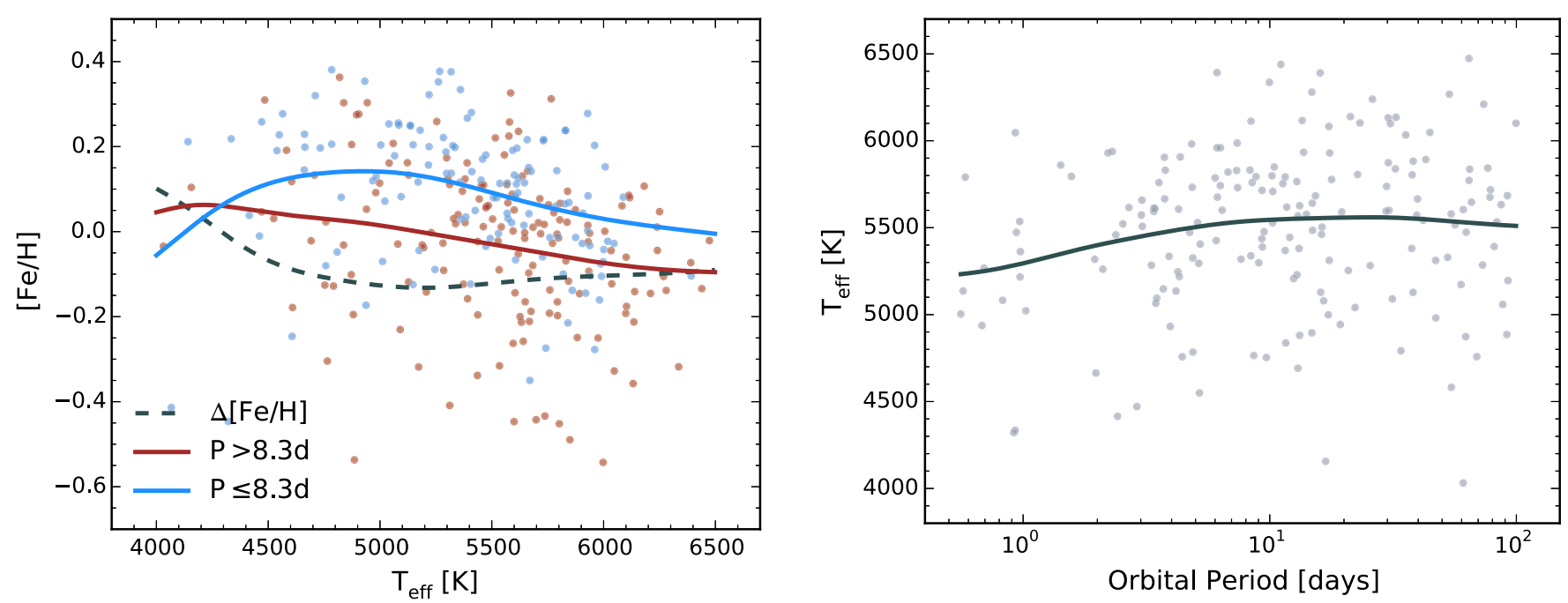

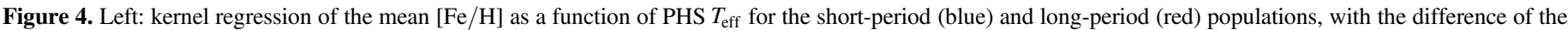

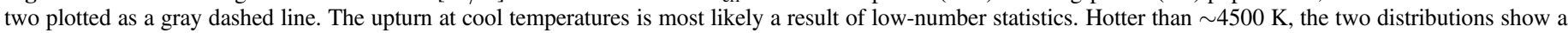

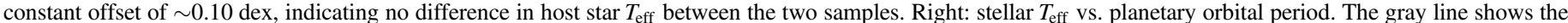

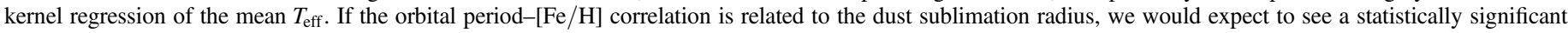
positive correlation in this plot, which we do not. Hence, there is no evidence for a dust sublimation effect.

by-product of rocky planet ingestion by the star driven by inward migration (e.g., Meléndez et al. 2009; Ramírez et al. 2010, 2014; Liu et al. 2014; Mack et al. 2014; Schuler et al. 2015; Teske et al. 2015, 2016a, 2016b; Bedell et al. 2017). For example, Mack et al. (2014) compared the elemental abundances of the twin stars in the planet-hosting wide binary system HD 20782/81. Both stars in that system host close-in planets within $\sim 0.2$ au, and Mack et al. (2014) found that both stars are significantly enriched in refractory elements. Those authors further used a model of planet ingestion to show that the enhanced refractory abundances were consistent with a scenario in which the observed close-in planet pushed 10-20 $M_{\oplus}$ of rocky material (perhaps in the form of small rocky planetesimals) into the surface convection zone of the star. Presumably, this is a consequence of the inward migration of the observed close-in planet. Thus, one possible explanation for the correlation we report here is that the planets in our sample that managed to migrate very close to their host stars ( $P<8.3$ days) were also more likely to shepherd other small rocky planets into the star, thus elevating its surface metallicity.

In theory, a testable prediction of this rocky material ingestion scenario would be a pattern of increasing stellar elemental abundances with elemental condensation temperature $\left(T_{c}\right)$, as found by Mack et al. (2014) (and, originally in solar twins, Meléndez et al. 2009). However, we are currently unable to perform this test owing to the uncertainties associated with dwarf star abundances derived by ASPCAP in DR14; as noted in Section 2, ASPCAP is optimized for red giants and not well tested for dwarfs. ${ }^{19}$ Moreover, while giant planet inward migration has been suggested as a mechanism for "pushing" refractory material onto the host star, we know of no studies suggesting that the inward migration of small planets would be capable of creating a similar abundance signature in their host stars.

\footnotetext{
${ }^{19}$ We did attempt to make a preliminary assessment of the differences between the slopes of $T_{c}$-abundance trends for the short- versus long-period samples within our 300 stars with planets, but we observed no difference between the slope distributions. If the short-period planet host stars had ingested more refractory material, we would expect them to show more positive slopes.
}

Interestingly, the scenario envisioned above could also potentially provide a natural explanation for the specific orbital period (8.3 days) dividing the metal-rich versus metal-poor samples. Previous work on the rotational evolution of premain-sequence stars has suggested a bimodal distribution of stellar rotation periods, the break between slow and rapid rotators occurring at $\sim 8$ days (see, e.g., Choi \& Herbst 1996, and references therein). The two groups of rotators have been interpreted by some authors as the result of different angular momentum histories, possibly due to rotational braking by those stars whose protoplanetary disks survive longer, draining angular momentum from the star via magnetic connection between star and disk at the co-rotation radius as noted above. If inward migration of planets, coupled with ingestion of rocky material by the star, is also related to the rapid dispersal of the protoplanetary disk, the result could be a natural division at $\sim 8$ days of those stars that were more likely to ingest refractory elements (i.e., show elevated surface metallicity) and more likely to host a close-in planet with an orbital period shorter than $\sim 8$ days.

If shorter-period planets usually orbit more rapidly rotating stars, based on our observed planet $P-[\mathrm{Fe} / \mathrm{H}]_{\text {star }}$ trend we would predict that higher-metallicity stars should show faster rotation and shorter disk lifetimes, on average. However, testing this prediction is complicated by the tendency for all stars to slow their rotation on gigayear timescales, regardless of their early rotational histories; we cannot know a priori the original rotation periods of the stars in our sample. To check whether their present-day stellar rotation periods show any trend with stellar metallicity or planetary orbital period, we cross-matched our sample with the McQuillan et al. (2013) and Walkowicz \& Basri (2013) catalogs of Kepler stellar rotation periods, resulting in 82 stars in common. In this subsample we see no trends between stellar rotation period and stellar metallicity or planet orbital period. Moreover, near-infrared observations of young stars in clusters of various metallicites find that lower-metallicity stars have shorter protoplanetary disk lifetimes (Yasui et al. 2009, 2010), and these observations are supported by models (Ercolano \& Clarke 2010) and 


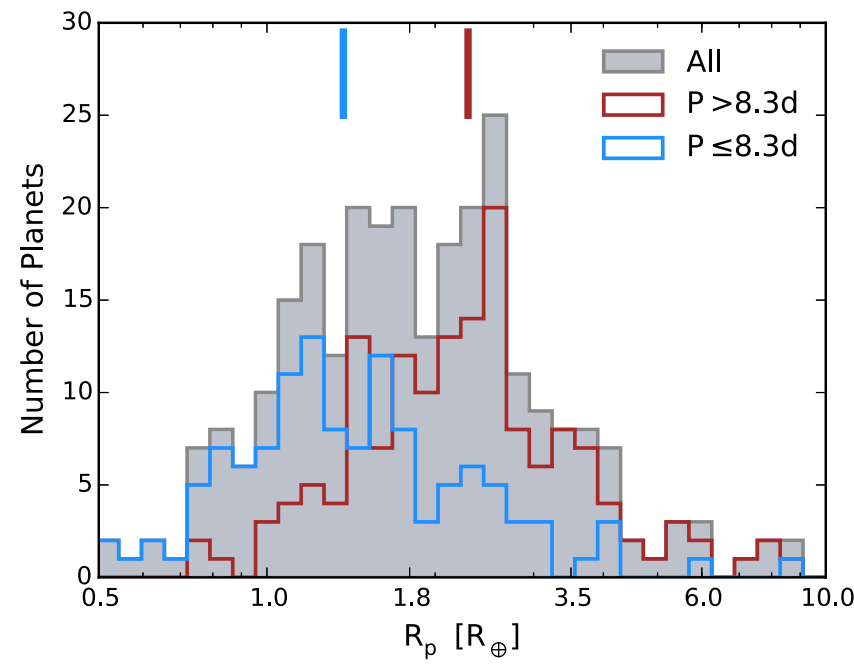

Figure 5. Histogram of KOI radii of the long-period (red) and short-period (blue) population, split by $P_{\text {crit }}=8.3$ days. The combined distribution is shown in gray. These are the same colors corresponding to the metal-enriched (blue) and solar-metallicity (red) distributions in the right panel of Figure 2. The tick marks at the top denote the median planet radius of the short-period (blue) and long-period (red) populations.

simulations (Nakatani et al. 2017) of protoplanetary disk evolution. This contradicts the prediction that shorter-period planets orbiting more metal-rich stars can be explained by shorter disk lifetimes (and by extension faster stellar rotation periods). Additionally, the idea of bimodal rotation rates among young stars has itself been disputed (see, e.g., Stassun et al. 1999).

Thus, we conclude that, while it is still plausible that the main trend we have discovered here could be the result of ingestion by stars of rocky material due to the inward migration of planets, it does not appear to be a consequence of star-disk interaction in the context of stellar rotational evolution.

\subsection{Planet Radius as a Third Dimension of the Correlation}

Specifically, Mulders et al. (2016) find an occurrence-corrected $\Delta[\mathrm{Fe} / \mathrm{H}]$ between planets interior and exterior to 10 days that varies with planetary radius, from $\Delta[\mathrm{Fe} / \mathrm{H}]=0.25 \pm 0.07 \mathrm{dex}$ for $R_{p}<1.7 R_{\oplus}$ planets to $0.08 \pm 0.05 \mathrm{dex}$ for $1.7 R_{\oplus} \leqslant R_{p}<$ $3.9 R_{\oplus}$ planets to $0.10 \pm 0.12 \mathrm{dex}$ for $R_{p} \geqslant 3.9 R_{\oplus}$ planets. We also find that the short-period (metal-rich host star) planets in our sample are statistically smaller $\left(p_{m w}=7.1 \sigma, p_{\mathrm{ks}}=6.7 \sigma\right)$ than the planets at longer periods (around less metal-rich stars). The median, mean, and standard deviation of our metal-rich/shortperiod planet population are $1.37,2.01$, and $1.91 R_{\oplus}$, respectively, versus $2.29,2.74$, and $1.91 R_{\oplus}$, respectively, in our solarmetallicity/long-period planet population; typical errors on $R_{p}$ are $0.02 R_{\oplus}$. Interestingly, the break in $[\mathrm{Fe} / \mathrm{H}]$-period space in our sample also appears to coincide with the reported "radius gap" around $1.8 R_{\oplus}$ defined by Fulton et al. (2017) (see Figure 5). While metal-poor dwarf stars will generally have larger radii than metal-rich dwarf stars, potentially influencing any trends with $R_{p}$ and host star $[\mathrm{Fe} / \mathrm{H}]$ (Gaidos \& Mann 2013), the metallicity bias (the difference between $[\mathrm{Fe} / \mathrm{H}]$ values of underlying population of stars versus those around which transiting planets are detected) for the stars in our sample is $\lesssim 0.02$ dex, below our measured precision. We will further explore this break in the $R_{p}-[\mathrm{Fe} / \mathrm{H}]-$ orbital period distribution within the APOGEE Kepler sample in an upcoming publication.
Recently, Owen \& Wu (2017) constructed a relatively simple analytical model, building on their previous numerical models (Owen \& Wu 2013), of a low-mass planet-core with a gas envelope-and how it changes with time under the influence of its host star flux. As their previous results (as well as those of Lopez \& Fortney 2013) showed, the radius distribution observed by Fulton et al. (2017) is matched by an "evaporation valley," such that smaller radius planets represent the bare cores of planets that have had their $\mathrm{H} / \mathrm{He}$ envelopes photoevaporated within the first $100 \mathrm{Myr}$. Owen \& $\mathrm{Wu}$ (2017) show specifically that the two peaks in $R_{p}$ observed by Fulton et al. arise because the timescale for mass loss is longest when the planet's radius has doubled in size owing to an accreted volatile envelope of approximately a few percent of the total planet mass. Their model is only able to reproduce the observed results when the planet envelopes are composed of primordial $\mathrm{H} / \mathrm{He}$, not water, and when the cores are roughly Earth-like in composition $\left(\rho \sim 5.5 \mathrm{~g} \mathrm{~cm}^{-3}\right)$. In summary, Owen \& Wu (2017) show that the small, short-period Kepler planets likely form from one parent population, with one average composition, and have a bimodal period-radius distribution due to envelope evaporation.

Almost simultaneous with Owen \& Wu (2017), Jin \& Mordasini (2017) produced work independent of Owen \& Wu (2017) that compared theoretical models of planet formation, thermodynamical evolution, and atmospheric escape of rockycored versus icy-cored planets with the results of Fulton et al. (2017). Similarly, the goal of Jin \& Mordasini (2017) was to understand better, in a statistical sense, how evaporation depends on planetary bulk composition. Jin \& Mordasini (2017) suggest-assuming that the radius gap is due to atmospheric evaporation - that small, short-period planets have mostly rocky cores made of silicates and iron, not mostly icy cores made of frozen $\mathrm{H}_{2} \mathrm{O}, \mathrm{CO}_{2}, \mathrm{CH}_{4}$, and/or $\mathrm{NH}_{3}$. Since planets with mostly icy cores can only form beyond the snow line, this indicates that close-in low-mass planets accreted mainly within the snow line, even considering migration (that is, migration must have been within the snow line). Classifying observed planets based on their ice mass fractions (derived from the mass and radius and an internal structure model; Mordasini et al. 2012b), as well as the planet's $R_{p}$ and semimajor axis $a$, the authors find eight categories of planets (see their Figures 6 and 7) that exhibit a clear compositional gradient with increasing planet radius. Interestingly, six of the eight planet categories requiring a rock-dominated composition $\left(R_{p} \lesssim 1.6 R_{\oplus}\right)$ are found within $\sim 0.09$ au $(\sim 10$ days around a solar-mass star), and all eight of the rock-dominated planets are found within $\sim 0.17$ au ( $\sim 26$ days around a solar-mass star). We see a second, less significant dip at $P \sim 23$ days in Figure 2, perhaps corresponding to this second rocky planet orbital period limit.

Putting this all together, the following picture emerges: most short-period, small planets have rocky cores, but their size, volatile content, and thus density is (in a general sense) tied to their orbital period and, as found in this study, their host star metallicity. The trend we see predicts that planets with little to no volatiles, and thus the smallest $R_{p}$ values, should be in the closest orbits and thus around more metal-rich host stars. Jin \& Mordasini (2017) caution that they do not see convincing evidence of a positive volatile content gradient with increasing semimajor axis, but that this is expected for evaporation. We note that K-S and A-D tests do not reveal a significant 
difference between the orbital periods of the 25 Type 1 and 3 planets of Jin \& Mordasini (2017) and their seven Type 6 planets (from their Table 1), but that with a larger number of small planets with well-constrained masses and radii, we can better test our prediction. Our results, as interpreted in the context of Jin \& Mordasini's theoretical framework, suggest that in the hunt for small, rock-dominated planets with little to no gaseous envelopes, one should be looking around more metal-rich stars.

\section{Conclusions}

In this work, we aim to characterize the intricate relationship between host star metallicity and planet orbital period, as it relates to the context of planet formation. We first demonstrate the veracity of ASPCAP's metallicities by comparing a sample of 221 FGK dwarfs in the APOGEE survey that also have quality parameters via optical spectroscopy in the literature. Then, using a sample of 282 short-period ( $P<100$ days) Kepler exoplanets and exoplanet candidates observed by the APOGEE KOI goal program and the associated parameters derived from ASPCAP, we have characterized the correlation between planet orbital period and host star metallicity. In particular, we have found the following:

1. There is a statistically significant correlation between host star $[\mathrm{Fe} / \mathrm{H}]$ and planetary orbital period that is characterized by a critical period, $P_{\text {crit }}=8.3_{-4.1}^{+0.1}$ days, below which planets preferentially orbit more metal-rich stars. This corresponds to a semimajor axis of $\sim 0.07$ au for a solarmass star and is consistent with the drop in occurrence rate at $\sim 0.1$ au found by Mulders et al. (2015b).

2. The minimum precision in $[\mathrm{Fe} / \mathrm{H}]$ needed to see this trend within our carefully vetted sample is $\sim 0.1$ dex. While this correlation has been seen in other studies (e.g., Mulders et al. 2016), the precision in APOGEE's abundance determinations allows us to find this correlation with higher confidence levels in a significantly smaller sample than what has been used for other planet host surveys of similar scale.

3. Planets in the short-period/high-metallicity population have significantly smaller radii than the long-period population $\left(p_{m w}=7.1 \sigma\right)$. Based on previous work on the "evaporation valley," this suggests that the population of planets around more metal-rich stars is mostly rocky and lacking substantial atmospheres, while the population of planets around more metal-poor stars have thicker atmospheres. Thus, to optimize the number of close-in, rocky exoplanets discovered around FGK dwarfs, transit surveys should prioritize supersolar-metallicity stars.

4. Based on the results of Jin \& Mordasini (2017), we suspect that the critical period of 8.3 days may be tied to the bulk composition of the exoplanet population in a statistical sense. In addition to this period, we find some evidence for a second, less convincing critical period at $P \sim 23$ days, which may also correlate with the exoplanet population's composition. Although we do not currently believe that this period is significant, its agreement with the results of Jin \& Mordasini (2017) is intriguing enough to warrant further investigation.

5. We hypothesize that there is some protoplanetary disk inner radius with a metallicity dependence at the time of planet formation that allows small, rocky planets to either form or migrate closer in to their host star in metal-rich conditions. Such an inner radius may be the dust sublimation radius, but we would expect this radius and thus orbital period to correlate strongly with the host $T_{\text {eff }}$, and we see no such correlation. The inner radius may instead be the gas co-rotation radius, but with our given observations it is hard to assess the likelihood of this explanation. Alternatively, the period-metallicity correlation that we observe may be the result of rocky planet ingestion, driven by inward planet migration. In this scenario, planets migrate inward and in the process shepherd rocky material (perhaps in the form of planetesimals) onto their host star, resulting in an increased surface metallicity. At this time the precision of APOGEE dwarf star abundances across a range of condensation temperatures precludes a robust test of this hypothesis.

APOGEE provides a valuable resource for characterizing exoplanet host stars from the Kepler mission. In particular, studies of planetary architecture coupled with accurate metallicities, as presented here, can provide new constraints on planet formation that could not otherwise be obtained from smaller, more focused studies. In addition, APOGEE measures $[\mathrm{Fe} / \mathrm{H}]$ to a level of precision that stands out from other spectroscopic surveys of similar scale, positioning APOGEE in a valuable area for future work in characterizing exoplanets and their host stars.

We would like to thank the anonymous referee, whose insightful comments improved the quality of our work and helped to find a bug in our code.

R.F.W., S.R.M., N.T., and M.F.S. were partially supported by the National Science Foundation under grant no. AST1616636. V.S. and K.C. acknowledge that their work here is supported, in part, by the National Aeronautics and Space Administration under grant 16-XRP16_2-0004, issued through the Astrophysics Division of the Science Mission Directorate. D.A.G.-H. was funded by the Ramón y Cajal fellowship no. RYC-2013-14182, and D.A.G.-H. and OZ acknowledge support provided by the Spanish Ministry of Economy and Competitiveness (MINECO) under grant AYA-2014-58082-P.

This research has made use of the NASA Exoplanet Archive, which is operated by the California Institute of Technology, under contract with the National Aeronautics and Space Administration under the Exoplanet Exploration Program. This research has also made use of the Tool for OPerations on Catalogs And Tables (Taylor 2005).

Funding for the Sloan Digital Sky Survey IV has been provided by the Alfred P. Sloan Foundation, the U.S. Department of Energy Office of Science, and the Participating Institutions. SDSS-IV acknowledges support and resources from the Center for High-Performance Computing at the University of Utah. The SDSS website is www.sdss.org. SDSS-IV is managed by the Astrophysical Research Consortium for the Participating Institutions of the SDSS Collaboration, including the Brazilian Participation Group, the Carnegie Institution for Science, Carnegie Mellon University, the Chilean Participation Group, the French Participation Group, Harvard-Smithsonian Center for Astrophysics, Instituto de Astrofísica de Canarias, Johns Hopkins University, Kavli Institute for the Physics and Mathematics of the Universe (IPMU)/University of Tokyo, Lawrence Berkeley National 
Laboratory, Leibniz Institut für Astrophysik Potsdam (AIP), Max-Planck-Institut für Astronomie (MPIA Heidelberg), MaxPlanck-Institut für Astrophysik (MPA Garching), Max-PlanckInstitut für Extraterrestrische Physik (MPE), National Astronomical Observatory of China, New Mexico State University, New York University, University of Notre Dame, Observatário Nacional/MCTI, The Ohio State University, Pennsylvania State University, Shanghai Astronomical Observatory, United Kingdom Participation Group, Universidad Nacional Autónoma de México, University of Arizona, University of Colorado Boulder, University of Oxford, University of Portsmouth, University of Utah, University of Virginia, University of Washington, University of Wisconsin, Vanderbilt University, and Yale University.

Facilities: Sloan, Kepler.

Software: Numpy (Van Der Walt et al. 2011), FERRE (Allende Prieto et al. 2006), ASPCAP (García Pérez et al. 2016).

\section{ORCID iDs}

Steven R. Majewski iㅣ https://orcid.org/0000-00032025-3147

Suvrath Mahadevan (1) https://orcid.org/0000-00019596-7983

Nicholas Troup (1) https://orcid.org/0000-0003-3248-3097

Keivan G. Stassun (i) https://orcid.org/0000-0002-3481-9052

\section{References}

Abolfathi, B., Aguado, D. S., Aguilar, G., et al. 2017, arXiv:1707.09322 Adibekyan, V. Z., Figueira, P., Santos, N. C., et al. 2013, A\&A, 560, A51 Adibekyan, V. Z., Sousa, S. G., Santos, N. C., et al. 2012, A\&A, 545, A32 Akeson, R. L., Christiansen, J., Ciardi, D. R., et al. 2017, in AAS Meeting 229 Abstracts, 146.16

Alibert, Y., Mordasini, C., \& Benz, W. 2011, A\&A, 526, A63

Allende Prieto, C., Beers, T. C., Wilhelm, R., et al. 2006, ApJ, 636, 804 Batalha, N. M. 2014, PNAS, 111, 12647

Beaugé, C., \& Nesvorný, D. 2013, ApJ, 763, 12

Bedell, M., Bean, J. L., Meléndez, J., et al. 2017, ApJ, 839, 94

Bertran de Lis, S., Allende Prieto, C., Majewski, S. R., et al. 2016, A\&A, 590, A74

Blanton, M. R., Bershady, M. A., Abolfathi, B., et al. 2017, arXiv:1703.00052

Borucki, W. J. 2016, RPPh, 79, 036901

Borucki, W. J., Koch, D., Basri, G., et al. 2010, Sci, 327, 977

Bouvier, J. 2013, in EAS Publications Ser. 62 Role and Mechanisms of Angular Momentum Transport During the Formation and Early Evolution of Stars, ed. P. Hennebelle \& C. Charbonnel (Les Ulis: EDP Sciences), 143

Brewer, J. M., Fischer, D. A., Valenti, J. A., \& Piskunov, N. 2016, ApJS, 225, 32

Brown, T. M., Latham, D. W., Everett, M. E., \& Esquerdo, G. A. 2011, AJ, 142,112

Bruntt, H., Basu, S., Smalley, B., et al. 2012, MNRAS, 423, 122

Bruntt, H., Bedding, T. R., Quirion, P.-O., et al. 2010, MNRAS, 405, 1907

Buchhave, L. A., Bizzarro, M., Latham, D. W., et al. 2014, Natur, 509, 593

Buchhave, L. A., \& Latham, D. W. 2015, ApJ, 808, 187

Buchhave, L. A., Latham, D. W., Johansen, A., et al. 2012, Natur, 486, 375

Burke, C. J., Christiansen, J. L., Mullally, F., et al. 2015, ApJ, 809, 8

Choi, P. I., \& Herbst, W. 1996, AJ, 111, 283

Cui, X.-Q., Zhao, Y.-H., Chu, Y.-Q., et al. 2012, RAA, 12, 1197

Dawson, R. I., Chiang, E., \& Lee, E. J. 2015, MNRAS, 453, 1471

Dawson, R. I., \& Murray-Clay, R. A. 2013, ApJL, 767, L24

Dong, S., Xie, J.-W., Zhou, J.-L., Zheng, Z., \& Luo, A. 2017, arXiv:1706. 07807

Dong, S., Zheng, Z., Zhu, Z., et al. 2014, ApJL, 789, L3

Dressing, C. D., \& Charbonneau, D. 2013, ApJ, 767, 95

Eisenstein, D. J., Weinberg, D. H., Agol, E., et al. 2011, AJ, 142, 72

Eisner, J. A., Hillenbrand, L. A., White, R. J., Akeson, R. L., \& Sargent, A. I. 2005, ApJ, 623, 952

Ercolano, B., \& Clarke, C. J. 2010, MNRAS, 402, 2735

Everett, M. E., Howell, S. B., Silva, D. R., \& Szkody, P. 2013, ApJ, 771, 107
Fleming, S. W., Mahadevan, S., Deshpande, R., et al. 2015, AJ, 149, 143 Fulton, B. J., Petigura, E. A., Howard, A. W., et al. 2017, arXiv:1703.10375 Gaidos, E., \& Mann, A. W. 2013, ApJ, 762, 41

García Pérez, A. E., Allende Prieto, C., Holtzman, J. A., et al. 2016, AJ, 151,144

Gautier, T. N., Borucki, W. J., Caldwell, D. A., \& Koch, D. G. 2007, in ASP Conf. Ser. 366, Transiting Extrapolar Planets Workshop, ed. C. Afonso, D. Weldrake, \& T. Henning (San Francisco, CA: ASP), 219

Ghezzi, L., Cunha, K., Smith, V. V., et al. 2010, ApJ, 720, 1290

Gonzalez, G. 1998, A\&A, 334, 221

Gunn, J. E., Siegmund, W. A., Mannery, E. J., et al. 2006, AJ, 131, 2332

Gustafsson, B., Edvardsson, B., Eriksson, K., et al. 2008, A\&A, 486, 951

Heiter, U., \& Luck, R. E. 2003, AJ, 126, 2015

Holtzman, J. A., Shetrone, M., Johnson, J. A., et al. 2015, AJ, 150, 148

Howard, A. W., Marcy, G. W., Bryson, S. T., et al. 2012, ApJS, 201, 15

Huber, D., Chaplin, W. J., Christensen-Dalsgaard, J., et al. 2013, ApJ, 767, 127

Ida, S., \& Lin, D. N. C. 2004, ApJ, 616, 567

Jin, S., \& Mordasini, C. 2017, arXiv:1706.00251

Kaufer, A., Stahl, O., Tubbesing, S., et al. 1999, Msngr, 95, 8

Kendall, M. G. 1938, Biometrika, 30, 81

Kennedy, G. M., \& Kenyon, S. J. 2008, ApJ, 673, 502

Koch, D. G., Borucki, W. J., Basri, G., et al. 2010, ApJL, 713, L79

Kuchner, M. J., \& Lecar, M. 2002, ApJL, 574, L87

Kurucz, R. L. 1993, in ASP Conf. Ser. 44, IAU Coll. 138, Peculiar versus

Normal Phenomena in A-type and Related Stars, ed. M. M. Dworetsky,

F. Castelli, \& R. Faraggiana (San Francisco, CA: ASP), 87

Liu, F., Asplund, M., Ramírez, I., Yong, D., \& Meléndez, J. 2014, MNRAS, 442, L51

Liu, F., Asplund, M., Yong, D., et al. 2016, MNRAS, 463, 696

Lo Curto, G., Mayor, M., Benz, W., et al. 2010, A\&A, 512, A48

Lopez, E. D., \& Fortney, J. J. 2013, ApJ, 776, 2

Mack, C. E., III, Schuler, S. C., Stassun, K. G., \& Norris, J. 2014, ApJ, 787, 98 Majewski, S. R., Schiavon, R. P., Frinchaboy, P. M., et al. 2017, AJ, 154, 94 Majewski, S. R. \& APOGEE Team, \& APOGEE-2 Team 2016, AN, 337, 863

Mann, H. B., \& Whitney, D. R. 1947, Ann. Math. Statist., 18, 50

Martin, R. G., \& Livio, M. 2012, MNRAS, 425, L6

Mayor, M., Pepe, F., Queloz, D., et al. 2003, Msngr, 114, 20

McQuillan, A., Mazeh, T., \& Aigrain, S. 2013, ApJL, 775, L11

Meléndez, J., Asplund, M., Gustafsson, B., \& Yong, D. 2009, ApJL, 704, L66

Min, M., Dullemond, C. P., Kama, M., \& Dominik, C. 2011, Icar, 212, 416

Mordasini, C., Alibert, Y., Benz, W., Klahr, H., \& Henning, T. 2012a, A\&A, 541, A97

Mordasini, C., Alibert, Y., Georgy, C., et al. 2012b, A\&A, 547, A112

Mulders, G. D., Ciesla, F. J., Min, M., \& Pascucci, I. 2015a, ApJ, 807, 9

Mulders, G. D., Pascucci, I., \& Apai, D. 2015b, ApJ, 798, 112

Mulders, G. D., Pascucci, I., Apai, D., Frasca, A., \& Molenda-Żakowicz, J. 2016, AJ, 152, 187

Mullally, F., Coughlin, J. L., Thompson, S. E., et al. 2015, ApJS, 217, 31

Muzerolle, J., Calvet, N., Hartmann, L., \& D'Alessio, P. 2003, ApJL, 597, L149

Nadaraya, E. A. 1964, Theory of Probability and its Applications, 9, 141

Najita, J. R., Carr, J. S., Glassgold, A. E., \& Valenti, J. A. 2007, in Protostars and Planets V, ed. B. Reipurth, D. Jewitt, \& K. Keil (Tucson, AZ: Univ. Arizona Press), 507

Nakatani, R., Hosokawa, T., Yoshida, N., Nomura, H., \& Kuiper, R. 2017, arXiv: 1706.04570

Nidever, D. L., Holtzman, J. A., Allende Prieto, C., et al. 2015, AJ, 150, 173

Nissen, P. E., Chen, Y. Q., Carigi, L., Schuster, W. J., \& Zhao, G. 2014, A\&A, 568, A25

Owen, J. E., \& Wu, Y. 2013, ApJ, 775, 105

Owen, J. E., \& Wu, Y. 2017, ApJ, 847, 29

Petigura, E. A., Howard, A. W., \& Marcy, G. W. 2013, PNAS, 110, 19273

Petigura, E. A., Howard, A. W., Marcy, G. W., et al. 2017, arXiv:1703.10400

Pinte, C., Ménard, F., Berger, J. P., Benisty, M., \& Malbet, F. 2008, ApJL, 673, L63

Plavchan, P., \& Bilinski, C. 2013, ApJ, 769, 86

Ramírez, I., Asplund, M., Baumann, P., Meléndez, J., \& Bensby, T. 2010, A\&A, 521, A33

Ramírez, I., Meléndez, J., \& Asplund, M. 2014, A\&A, 561, A7

Rein, H. 2012, MNRAS, 427, L21

Rice, W. K. M., \& Armitage, P. J. 2003, ApJL, 598, L55

Santos, N. C., Israelian, G., \& Mayor, M. 2004, A\&A, 415, 1153

Santos, N. C., Mayor, M., Bonfils, X., et al. 2011, A\&A, 526, A112

Schlaufman, K. C. 2015, ApJL, 799, L26

Schlaufman, K. C., \& Laughlin, G. 2011, ApJ, 738, 177

Schuler, S. C., Vaz, Z. A., Katime Santrich, O. J., et al. 2015, ApJ, 815, 5

Shetrone, M., Bizyaev, D., Lawler, J. E., et al. 2015, ApJS, 221, 24 
Silburt, A., Gaidos, E., \& Wu, Y. 2015, ApJ, 799, 180

Sneden, C. 1973, ApJ, 184, 839

Sousa, S. G., Santos, N. C., Israelian, G., Mayor, M., \& Monteiro, M. J. P. F. G. 2007, A\&A, 469, 783

Sousa, S. G., Santos, N. C., Mayor, M., et al. 2008, A\&A, 487, 373

Souto, D., Cunha, K., García-Hernández, D. A., et al. 2017, ApJ, 835, 239

Spearman, C. 1904, The American Journal of Psychology, 15, 72

Stassun, K. G., Mathieu, R. D., Mazeh, T., \& Vrba, F. J. 1999, AJ, 117, 2941

Taylor, M. B. 2005, in ASP Conf. Ser. 347, Astronomical Data Analysis Software and Systems XIV, ed. P. Shopbell, M. Britton, \& R. Ebert (San Francisco, CA: ASP), 29

Teske, J. K., Ghezzi, L., Cunha, K., et al. 2015, ApJL, 801, L10

Teske, J. K., Khanal, S., \& Ramírez, I. 2016a, ApJ, 819, 19

Teske, J. K., Shectman, S. A., Vogt, S. S., et al. 2016b, AJ, 152, 167

Troup, N. W., Nidever, D. L., De Lee, N., et al. 2016, AJ, 151, 85

Valenti, J. A., \& Fischer, D. A. 2005, ApJS, 159, 141

Valenti, J. A., \& Piskunov, N. 1996, A\&AS, 118, 595
Van Der Walt, S., Colbert, S. C., \& Varoquaux, G. 2011, arXiv:1102.1523

Vogt, S. S., Allen, S. L., Bigelow, B. C., et al. 1994, Proc. SPIE, 2198, 362

Walkowicz, L. M., \& Basri, G. S. 2013, MNRAS, 436, 1883

Wang, J., \& Fischer, D. A. 2015, AJ, 149, 14

Watson, G. S. 1964, Sankhyā Ser., 26, 359, http://www.jstor.org/stable/ 25049340?seq=1\#page_scan_tab_contents

Wilson, J. C., Hearty, F., Skrutskie, M. F., et al. 2010, Proc. SPIE, 7735, 77351C

Wilson, J. C., Hearty, F., Skrutskie, M. F., et al. 2012, in AAS Meeting 219 Abstracts, 428.02

Winn, J. N., Sanchis-Ojeda, R., Rogers, L., et al. 2017, arXiv:1704.00203

Xiao, L., Niu, R., \& Zhang, H. 2017, MNRAS, 467, 2869

Yadav, R. K. S., Bedin, L. R., Piotto, G., et al. 2008, A\&A, 484, 609

Yasui, C., Kobayashi, N., Tokunaga, A. T., Saito, M., \& Tokoku, C. 2009, ApJ, 705, 54

Yasui, C., Kobayashi, N., Tokunaga, A. T., Saito, M., \& Tokoku, C. 2010, ApJL, 723, L113

Zasowski, G., Johnson, J. A., Frinchaboy, P. M., et al. 2013, AJ, 146, 81 